\title{
On the Capacity of MIMO Broadcast Channels With Partial Side Information
}

\author{
Masoud Sharif, Student Member, IEEE, and Babak Hassibi
}

\begin{abstract}
In multiple-antenna broadcast channels, unlike point-to-point multiple-antenna channels, the multiuser capacity depends heavily on whether the transmitter knows the channel coefficients to each user. For instance, in a Gaussian broadcast channel with $M$ transmit antennas and $n$ single-antenna users, the sum rate capacity scales like $M \log \log n$ for large $n$ if perfect channel state information (CSI) is available at the transmitter, yet only logarithmically with $M$ if it is not.

In systems with large $n$, obtaining full CSI from all users may not be feasible. Since lack of CSI does not lead to multiuser gains, it is therefore of interest to investigate transmission schemes that employ only partial CSI. In this paper, we propose a scheme that constructs $M$ random beams and that transmits information to the users with the highest signal-to-noise-plus-interference ratios (SINRs), which can be made available to the transmitter with very little feedback. For fixed $M$ and $n$ increasing, the throughput of our scheme scales as $M \log \log n N$, where $N$ is the number of receive antennas of each user. This is precisely the same scaling obtained with perfect CSI using dirty paper coding. We furthermore show that a linear increase in throughput with $M$ can be obtained provided that $M$ does not not grow faster than $\log n$. We also study the fairness of our scheduling in a heterogeneous network and show that, when $M$ is large enough, the system becomes interference dominated and the probability of transmitting to any user converges to $\frac{1}{n}$, irrespective of its path loss. In fact, using $M=\alpha \log n$ transmit antennas emerges as a desirable operating point, both in terms of providing linear scaling of the throughput with $M$ as well as in guaranteeing fairness.
\end{abstract}

Index Terms-Broadcast channel, channel state information (CSI), multiuser diversity, wireless communications.

\section{INTRODUCTION}

$\mathbf{M}$ ULTIPLE-antenna communications systems have generated a great deal of interest since they are capable of considerably increasing the capacity of a wireless link. In fact, it was known for a long time that, if perfect channel state information (CSI) were available at the transmitter and receiver, then they could jointly diagonalize the channel, thereby creating as many parallel channels as the minimum of the number of transmit/receive antennas and thus increase the capacity of the channel by this same factor. More surprisingly, it was later shown that the same capacity scaling is true if the channel is not known at the transmitter [1], [2] and even if it is not known at the

Manuscript received June 25,2003; revised August 31, 2004. This work was supported in part by the National Science Foundation under Grant CCR-0133818, by the Office of Naval Research under Grant N00014-02-1-0578, and by the Lee Center for Advanced Networking at the California Institute of Technology.

The authors are with the Department of Electrical Engineering, California Institute of Technology, Pasadena, CA 91125 USA (e-mail: masoud@ systems. caltech.edu; hassibi@systems.caltech.edu).

Communicated by G. Caire, Associate Editor for Communications.

Digital Object Identifier 10.1109/TIT.2004.840897 receiver [3], [4] (provided the coherence interval of the channel is not too short).

While these are all true for point-to-point communications links, there has only been recent interest in the role of multiple-antenna systems in a multiuser network environment, and especially in broadcast and multiple-access scenarios. There has been a line of work studying scheduling algorithms in multiple-input multiple-output (MIMO) broadcast channels [5] with the main result being that, due to channel hardening in MIMO systems, many of the multiuser gains disappear. There has been another line of work studying the sum-rate capacity, and in fact, the capacity region, of MIMO broadcast channels [6]-[8]. It has been shown that the sum-rate capacity is achieved by dirty paper coding and, moreover recently, it has been shown that dirty paper coding in fact achieves the capacity region of the Gaussian MIMO broadcast channel [9].

While the above results suggest that capacity increases linearly in the number of transmit antennas, they all rely on the assumption that the channel is known perfectly at the transmitter. Moreover, the dirty paper coding scheme, especially in the multiuser context, is extremely computationally intensive (although suboptimal schemes such as channel inversion or Tomlinson-Harashima precoding [10]-[12] give relatively close performance to the optimal schemes). One may speculate whether, as in the point-to-point case, it is possible to get the same gains without having channel knowledge at the transmitter. Unfortunately, it is not too difficult to convince oneself that, if no channel knowledge is available at the transmitter, then using any conventional scheduling scheme, capacity scales only logarithmically in the number of transmit antennas. In fact, in this case, increasing the number of transmit antennas yields no gains since the same performance can be obtained with a single transmit antenna operating at higher power.

In many applications, however, it is not reasonable to assume that all the channel coefficient to every user can be made available to the transmitter. This is especially true if the number of transmit antennas $M$ and/or the number of users $n$ is large (or if the users are mobile and are moving rapidly). Since perfect CSI may be impractical, yet no CSI is useless, it is very important to devise and study transmission schemes that require only partial CSI at the transmitter. This is the main goal of the current paper.

The scheme we propose is one that constructs $M$ random orthonormal beams and transmits to users with the highest signal-to-noise-plus-interference ratios (SINRs). In this sense, it is in the same spirit as the work of [13] where the transmission of random beams is also proposed.

However, our scheme differs in several key respects. First, we send multiple beams (in fact, $M$ of them) whereas [13] sends 
only a single beam. Second, whereas the main concern in [13] is to improve the proportional fairness of the system (by giving different users more of a chance to be the best user) our scheme aims at capturing as much of the broadcast channel capacity as possible. Fairness ${ }^{1}$ is achieved in our system as a convenient by-product.

We should remark that our scheme requires far less feedback than one that provides full CSI. To have full channel knowledge at the receiver, each user must feed back $M$ complex numbers (its channel gains) to the transmitter. Here each user needs only to feed back one real number (its best SINR) and the corresponding index which is an integer number. In fact, it turns out that only users who have favorable SINRs need to do so, which can considerably reduce the amount of feedback required.

Based on asymptotic analysis, we show that, for fixed $M$ and $n$ increasing, our proposed scheme achieves a throughput ${ }^{2}$ of $M \log \log n N$, where $N$ is the number of receive antennas of each user. Happily, this is the same as the scaling law of the sum-rate capacity when perfect CSI is available [14], and so, asymptotically, our scheme does not suffer a loss in this regime. One may ask how fast may $M$ grow to guarantee a linear scaling of the throughput $R$ with $M$ ? We show that the answer is $M=$ $O(\log n):$ more precisely, if $\frac{M}{\log n} \rightarrow \alpha$ then $\frac{R}{M} \rightarrow \alpha^{\prime}$, whereas if $\frac{M}{\log n} \rightarrow \infty$ then $\frac{R}{M} \rightarrow 0$.

In schemes (such as ours) that exploit multiuser diversity there is often tension between increasing capacity (by transmitting to the strongest users) and fairness, the reason being that the strongest users (here meaning the users closest to the base station) may dominate the network. Fortunately, we show that in our scheme, provided the number of transmit antennas is large enough, the system becomes interference dominated and so, although close users receive strong signal they also receive strong interference. Therefore, it can be shown that, for large enough $M$ and in a heterogeneous network, the probability of any user having the highest SINR converges to $\frac{1}{n}$, irrespective of how strong their signal strength is. A more careful study of this issue reveals that the choice of $M=\alpha \log n$ transmit antennas is a desirable operating point, both in terms of providing linear scaling of the throughput with $M$ as well as in guaranteeing fairness.

The remainder of this paper is organized as follows. Section II describes the formulation of the problem. Our proposed scheduling algorithm is introduced in Section III. In Section IV, the asymptotic analysis of the throughput of our scheme is presented for the case where the number of users is increasing, $M$ (number of transmit antennas) is fixed, and each user has a single receive antenna $(N=1)$. Section $\mathrm{V}$ considers the case where $M$ is allowed to grow to infinity as well. In Section VI, different scenarios for $N>1$ is considered and the asymptotic behavior of their throughput is obtained. Fairness of our scheduling when the users have different SNRs is considered in Section VII. Section VIII presents the simulation result for the throughput and fairness of our proposed scheduling. Finally, Section IX concludes the paper.

\footnotetext{
${ }^{1}$ In this paper, by fairness we mean that the probability of choosing users with different SNRs is equal.

${ }^{2}$ In this paper, throughput refers to the achievable sum average rate by the scheduling scheme.
}

\section{PROBLEM FORMULATION}

In this paper, we consider a multiple-antenna Gaussian broadcast channel with $n$ receivers equipped with $N$ antennas and a transmitter with $M$ antennas. We consider the block-fading model for the channel described by a propagation matrix which is constant during the coherence interval of $T$. Since in a typical cellular system, the number of users is much larger than the number of transmit antennas and also the number of antennas in the base station (or the transmitter) is greater than the number of antennas in the receiver, we often assume $n \gg M$ and $N \leq M$ throughout the paper.

Let $S(t)$ be the $M \times 1$ vector of the transmit symbols at time slot $t$, and let $Y_{i}(t)$ be the $N \times 1$ vector of the received signal at the $i$ th receiver related by

$$
Y_{i}(t)=\sqrt{\rho_{i}} H_{i} S(t)+W_{i}, \quad i=1, \ldots, n
$$

where $H_{i}$ is an $N \times M$ complex channel matrix, known perfectly to the receiver, $W_{i}$ is an $N \times 1$ additive noise, and the entries of $H_{i}$ and $W_{i}$ are independent and identically distributed (i.i.d.) complex Gaussian with zero mean and unit variance $C N(0,1)$. Moreover, the total transmit power is assumed to be $M$, i.e., $E\left\{S^{*} S\right\}=M$; in other words, the transmit power per antenna is one. ${ }^{3}$ Therefore, the received SNR of the $i$ th user will be $E\left\{\rho_{i}\left|H_{i} S\right|^{2}\right\}=M \rho_{i}$, however, to simplify the notation we refer to $\rho_{i}$ as the SNR of the $i$ th user.

To analyze the throughput of the system, we consider a homogeneous network in which all the users have the same SNR, i.e., $\rho_{i}=\rho$ for $i=1, \ldots, n$. However, in the last part of the paper, we look into the fairness issue when the network is heterogeneous in which the users have different SNRs.

\section{SCHEDULING ALGORITHM USING RANDOM BEAMFORMING}

The capacity of point-to-point multiple-antenna systems has been investigated with different assumption for the CSI, whether the receiver/transmitter knows the channel or not. As it is shown in [1], [2], if the receiver knows the channel, the capacity scales like $\min (M, N) \log \rho$ no matter whether the transmitter knows the channel or not. Indeed, it is shown in [3], [4] that when the receiver does not know CSI, the capacity scales like

$$
\min (M, N)\left(1-\frac{\min (M, N)}{T}\right) \log \rho
$$

where $T$ is the coherence interval of the channel.

While the full CSI in the transmitter does not seem to be beneficial in the point-to-point communication, the knowledge of the channel is crucial in broadcast channel [5], [15]. For the case with the full CSI available at both the transmitter and the receivers, it is shown that the sum rate capacity of the Gaussian broadcast channel can be achieved by using dirty paper coding

\footnotetext{
${ }^{3}$ This is in contrast to the convention used in single-link MIMO channels where the total transmit power $E\{S * S\}=1$ is fixed. There this is done to make a fair comparison with a single-antenna channel operating at the same transmit power. Here, however, since we will be transmitting to $M$ different users, we would like to make a comparison to $M$ independent single-antenna links each operating at unit power. Hence, our normalization will be $E\{S * S\}=M$.
} 
[6]-[8]. More precisely, for the case where $N=1$, the sum rate capacity $R^{D P}$ can be written as

$$
R^{D P}=E\left\{\max _{\left\{P_{1}, \ldots, P_{n}, \sum P_{i}=M \rho\right\}} \log \operatorname{det}\left(1+\sum_{i=1}^{n} H_{i}^{*} P_{i} H_{i}\right)\right\}
$$

where $H_{i}$ is a $1 \times M$ channel matrix and $M \rho$ is the total average power. In Appendix B, the following lemma is proved.

Lemma 1: Suppose both the transmitter and receivers know the channel perfectly in a Gaussian broadcast channel with $n$ single-antenna receivers with average transmit power of $M \rho$, and the transmitter has $M$ antennas. Let also $M$ and $\rho$ be fixed, then for sufficiently large $n$, the sum rate capacity scales like $M \log \log n$.

Therefore, when the transmitter and receivers have full CSI, the sum rate capacity scales linearly with $M$. On the other hand, having full CSI in both sides requires a lot of feedback and practically it is unrealistic. This motivates the question of how much partial side information is needed in the transmitter that provides us a linear scaling of the throughput with $M$ and reduces the amount of feedback [5], [15], [16].

In this paper, in order to exploit having multiple antennas in the transmitter without having full CSI in the transmitter, we propose a scheme that constructs $M$ random beams and transmits to the users with the highest SINRs. For simplicity, we assume $N=1$ and we choose $M$ random orthonormal vectors $\phi_{m}(M \times 1)$ for $m=1, \ldots, M$ where $\phi_{i}$ 's are generated according to an isotropic distribution [4]. Then at time slot $t$, the $m$ th vector is multiplied by the $m$ th transmit symbol $s_{m}(t)$, so that the transmitted signal is

$$
S(t)=\sum_{m=1}^{M} \phi_{m}(t) s_{m}(t), \quad t=1, \ldots, T .
$$

Following our earlier assumption and using the independence of $s_{i}$ 's, the average transmit power per antenna is one, equivalently, $E\left\{\left|s_{i}\right|^{2}\right\}=1$, and henceforth the total transmit power is $E\left\{S^{*} S\right\}=M$. After $T$ channel uses, we independently choose another set of orthonormal vectors $\left\{\phi_{m}\right\}$, and so on. In this paper, we assume $s_{m}$ 's are letters from codewords of a Gaussian capacity-achieving codebook. We further assume that the coding is performed across several blocks.

From now on, for simplicity, we drop the time index from $S_{i}(t)$ and $Y_{i}(t)$, and therefore, the received signal at the $i$ th receiver is

$$
Y_{i}=\sum_{m=1}^{M} H_{i} \phi_{m} s_{m}+W_{i}, \quad i=1, \ldots, n .
$$

We assume that the $i$ th receiver knows $H_{i} \phi_{m}$ for $m=1, \ldots, M$ (this can be readily arranged by training). Therefore, the $i$ th receiver can compute the following $M$ SINRs by assuming that $s_{m}$ is the desired signal and the other $s_{i}$ 's are interference as follows:

$$
\operatorname{SINR}_{i, m}=\frac{\left|H_{i} \phi_{m}\right|^{2}}{1 / \rho+\sum_{k \neq m}\left|H_{i} \phi_{k}\right|^{2}}, \quad m=1, \ldots, M .
$$

Note that on average the SINRs behave like ${ }^{4}$

$$
\operatorname{SINR}_{i, m} \approx \frac{1}{1 / \rho+(M-1)} \approx \frac{1}{M-1} .
$$

Thus, if we randomly assign beams to users, the throughput will be

$$
\begin{aligned}
R & =E\left\{\sum_{i=1}^{M} \log \left(1+\operatorname{SINR}_{i, m}\right)\right\} \\
& =M E \log \left(1+\operatorname{SINR}_{i, m}\right) \\
& \leq M \log \left(1+E\left\{\operatorname{SINR}_{i, m}\right\}\right) \\
& \approx M \log \left(1+\frac{1}{M-1}\right)<\frac{M}{M-1} \approx 1 .
\end{aligned}
$$

Thus, even though we are sending $M$ different signals, we do not get an $M$-fold increase in the throughput. Therefore, the side information in the transmitter is crucial to exploit the multiuser diversity.

Suppose now each receiver feeds back its maximum SINR, i.e., $\max _{1 \leq m \leq M} \operatorname{SINR}_{i, m}$, along with the index $m$ in which the SINR is maximized. Therefore, in the transmitter, instead of randomly assigning each beam to one of the users, the transmitter assigns $s_{m}$ to the user with the highest corresponding SINR, i.e., $\max _{1 \leq i \leq n} \operatorname{SINR}_{i, m}$. So if we do the above scheduling, the throughput can be written roughly as

$$
\begin{aligned}
R & \approx E\left\{\sum_{m=1}^{M} \log \left(1+\max _{1 \leq i \leq n} \operatorname{SINR}_{i, m}\right)\right\} \\
& =M E\left\{\log \left(1+\max _{1 \leq i \leq n} \operatorname{SINR}_{i, m}\right)\right\}
\end{aligned}
$$

where we used " $\approx$ " instead of " $=$ " since there is a small probability that user $i$ may be the strongest user for more than one signal $s_{m}$. In Section IV, we shall see that this is very unlikely as $n$ increases, and so the above approximation approaches equality.

It is important to note that compared to (6), we have a maximization over $i$ inside the logarithm. Thus, we need to study the distribution of $\max _{1 \leq i \leq n} \operatorname{SINR}_{i, m}$ which as we shall see, has a huge effect on the end result. We also remark that, as we shall see in Section IV, it is not even necessary for all users to send back their strongest SINR, which considerably reduces the required feedback.

\section{ASYMPTOTIC ANALYSIS OF THE ThroughPUT: $N=1, M$ Is FIXED}

In this section, we obtain lower and upper bounds for the throughput when $M$ is fixed, $N=1$, and $n$ is going to infinity. Using $M$ random beams and sending to the users with the highest SINRs, we can bound the throughput $R$ as

$$
R \leq E\left\{\sum_{m=1}^{M} \log \left(1+\max _{i=1, \ldots, n} \operatorname{SINR}_{i, m}\right)\right\}
$$

where this is an upper bound since we ignored the probability that user $i$ be the maximum SINR user twice (if this is the case, the transmitter has to choose another user with SINR less than

\footnotetext{
${ }^{4}$ This can be made more precise, however, for the sake of brevity we just mention a sketchy argument.
} 
the maximum SINR which therefore decreases the throughput). On the other hand, the following lemma states a lower bound for the throughput as well.

Lemma 2: Let $R$ be the throughput of the random beamforming scheduling. Then

$$
\begin{aligned}
& R \geq M\left(1-\left\{\operatorname{Pr}\left\{\operatorname{SINR}_{i, 1} \leq 1\right\}\right\}^{n}\right) \\
& \quad \cdot E\left\{\log \left(1+\max _{i=1, \ldots, n} \operatorname{SINR}_{i, 1}\right)||_{i=1, \ldots, n} \operatorname{SINR}_{i, 1} \geq 1\right\} .
\end{aligned}
$$

Proof: First of all, we make the following observation: for any $r \in\{1, \ldots, M\}$, conditioning on the fact that

$$
\operatorname{SINR}_{j, r}=\max _{1 \leq i \leq n} \operatorname{SINR}_{i, r} \geq 1
$$

then $\operatorname{SINR}_{j, r}$ has to be the maximum over $m=1, \ldots, M$ as well, i.e.,

$$
\operatorname{SINR}_{j, r}=\max _{m=1, \ldots, M} \operatorname{SINR}_{j, m}
$$

This can be easily proved as follows; assuming $\operatorname{SINR}_{j, r}>1$, we have

$$
\left|H_{j} \phi_{r}\right|^{2} \geq 1 / \rho+\sum_{k \neq r}\left|H_{j} \phi_{k}\right|^{2} \geq\left|H_{j} \phi_{m}\right|^{2}, \quad m=1, \ldots, M .
$$

Now we can write $\operatorname{SINR}_{j, m}$ and $m \neq r$ as

$$
\operatorname{SINR}_{j, m}=\frac{\left|H_{j} \phi_{m}\right|^{2}}{1 / \rho+\sum_{k \neq m}\left|H_{j} \phi_{k}\right|^{2}}<\frac{\left|H_{j} \phi_{m}\right|^{2}}{\left|H_{j} \phi_{r}\right|^{2}} \leq 1
$$

and hence, $\operatorname{SINR}_{i, r}$ is the maximum over $m=1 \ldots, M$ as well, i.e.,

$$
\operatorname{SINR}_{i, r}=\max _{m=1, \ldots, M} \operatorname{SINR}_{i, m} .
$$

Therefore, it is impossible for a user to be the maximum SINR for two signals conditioning on the fact that $\max _{1 \leq i \leq n} \operatorname{SINR}_{i, r} \geq 1$. Thus, the throughput can be bounded as

$$
\begin{aligned}
& R \geq \sum_{m=1}^{M} \operatorname{Pr}\left\{\max _{i=1, \ldots, n} \operatorname{SINR}_{i, m} \geq 1\right\} \\
& \cdot E\left\{\log \left(1+\max _{i=1, \ldots, n} \operatorname{SINR}_{i, m}\right) \mid \max _{i=1, \ldots, n} \operatorname{SINR}_{i, m} \geq 1\right\} .
\end{aligned}
$$

Since $\Phi=\left(\phi_{1} \ldots \phi_{M}\right)$ is a unitary matrix, so $H_{i} \Phi$ is a vector with i.i.d. $C N(0,1)$ entries. This implies that $\left|H_{i} \phi_{m}\right|^{2}$ are i.i.d. over $m$ (and also over $i$ ) with $\chi^{2}(2)$ distribution. Therefore, $\operatorname{SINR}_{i, m}$ for $i=1, \ldots, n$ are i.i.d. but not independent over $m=1, \ldots, M$. Thus,

$$
\operatorname{Pr}\left\{\max _{i=1, \ldots, n} \operatorname{SINR}_{i, m} \leq 1\right\}=\left(\operatorname{Pr}\left\{\operatorname{SINR}_{i, 1} \leq 1\right\}\right)^{n} .
$$

Substituting (12) in (11) completes the proof.

As we shall show later, the lower and upper bounds for the throughput become tight for sufficiently large $n$ and when $\lim _{n \rightarrow \infty} \frac{M}{\log n}=0$. In this case, conditioning on $\max _{1 \leq i \leq M} \operatorname{SINR}_{i, m} \geq 1$ in Lemma 2 can be replaced by $\max _{1 \leq i \leq M} \operatorname{SINR}_{i, m} \geq \eta$ where $\eta$ is a constant independent of $n$ and the bounds remain tight. This implies that the receiver is only required to feed back its maximum SINR if it is greater than $\eta$ along with the index $m$ corresponding to the signal. Therefore, the amount of feedback here will be $n \operatorname{Pr}\left\{\max _{1 \leq m \leq M} \operatorname{SINR}_{i, m} \geq \eta\right\}$ real numbers and $M$ integers (at most). However, in the case with full CSI in the transmitter, the amount of feedback is $2 n M$ real numbers which is roughly $2 M$ times bigger than what we need in our scheme. Furthermore, the complexity of our scheme is much less than the proposed schemes to implement dirty paper coding with full CSI using nested lattices or trellis precoding [10], [11].

In order to evaluate the lower and upper bounds, we have to obtain the distribution of $\operatorname{SINR}_{i, m}$. As mentioned earlier, $\left|H_{i} \phi_{m}\right|^{2}$ 's are i.i.d. over $m$ (and also over $i$ ) with $\chi^{2}(2)$ distribution. Thus,

$$
\operatorname{SINR}_{i, m}=\frac{\left|H_{i} \phi_{m}\right|^{2}}{1 / \rho+\sum_{k \neq m}\left|H_{i} \phi_{k}\right|^{2}}=\frac{z}{1 / \rho+y}
$$

where $z$ has $\chi^{2}(2)$, and $y$ has $\chi^{2}(2 M-2)$ distributions (denoted by $f_{Y}(\cdot)$ ). Conditioning on $y$, the probability distribution function (pdf) of $\operatorname{SINR}_{i, m}, f_{s}(x)$, can be written as

$$
\begin{aligned}
f_{s}(x) & =\int_{0}^{\infty} f_{s \mid Y}(x \mid y) f_{Y}(y) d y \\
& =\int_{0}^{\infty}(1 / \rho+y) e^{-(1 / \rho+y) x} \times \frac{y^{M-2} e^{-y}}{(M-2) !} d y \\
& =\frac{e^{-x / \rho}}{(1+x)^{M}}\left(\frac{1}{\rho}(1+x)+M-1\right) .
\end{aligned}
$$

We can also calculate the cumulative distribution function (cdf) of $\operatorname{SINR}_{i, m}, F_{s}(x)$, as

$$
\begin{aligned}
F_{s}(x) & =\int_{0}^{x} \frac{e^{-x / \rho}}{(1+x)^{M}}\left(\frac{1}{\rho}(1+x)+M-1\right) d x \\
& =1-\frac{e^{-x / \rho}}{(1+x)^{M-1}}, \quad x \geq 0 .
\end{aligned}
$$

Since $\operatorname{SINR}_{i, m}$ for $i=1, \ldots, n$ are i.i.d. random variables, the cdf of $\max _{1 \leq i \leq n} \operatorname{SINR}_{i, m}$ for $m=1 \ldots, M$ is $\left(F_{s}(x)\right)^{n}$. Using the obtained cdf we can now evaluate the throughput of our proposed randomly chosen beam-forming technique.

Lemma 3: For any $\rho, M$, and $n$, the throughput of the randomly chosen beamforming satisfies

$$
\begin{aligned}
& M \int_{1}^{\infty} \log (1+x) n f_{s}(x) F_{s}^{n-1}(x) d x \\
& \quad \leq R \leq M \int_{0}^{\infty} \log (1+x) n f_{s}(x) F_{s}^{n-1}(x) d x
\end{aligned}
$$

where $f_{s}(x)$ and $F_{s}(x)$ are as defined in (14) and (15), respectively.

Proof: The upper bound clearly follows from (8) by substituting the distribution of the maximum SINR in (8). To prove the lower bound, we can write the conditional distribution of $\max _{1 \leq i \leq n} \operatorname{SINR}_{i, 1}$ given that $\max _{1 \leq i \leq n} \operatorname{SINR}_{i, 1} \geq 1$ as

$$
\begin{aligned}
\operatorname{Pr}\left\{\max _{i=1, \ldots, n} \operatorname{SINR}_{i, 1}<a \mid \max _{i=1, \ldots, n} \operatorname{SINR}_{i, 1}>1\right\} & \\
& = \begin{cases}\frac{F_{s}^{n}(a)-F_{s}^{n}(1)}{1-F_{s}^{n}(1)}, & a \geq 1 \\
0, & a \leq 1 .\end{cases}
\end{aligned}
$$


Now taking the derivative of the cdf, and substituting the pdf in (9), we can derive the lower bound as stated in (16).

Lemma 3 can be used to evaluate the throughput for any $n$, $\rho$, and $M$. However, in many systems, $\rho$ and $M$ are fixed, but $n$ (the number of users) is large. It is therefore useful to investigate this regime. In what follows, we will focus on the scaling laws of the throughput for large $n$.

In fact, the asymptotic behavior of the distribution of the maximum of $n$ i.i.d. random variables has been extensively studied in the literature [17]-[19]. In Appendix A, we review results that we need in this paper. Corollary A.1 in Appendix A can be used to state the following result.

Lemma 4: Let $\mathrm{SINR}_{i, m}, i=1, \ldots, n$, be $n$ i.i.d. random variables with distribution function $f_{s}(x)$ as in (14). Then, for $M$ and $\rho$ fixed and $n$ sufficiently large

$$
\begin{aligned}
& \operatorname{Pr}\{\rho \log n-\rho M \log \log n+O(\log \log \log n) \\
& \leq \max _{1 \leq i \leq n} \operatorname{SINR}_{i, m} \leq \rho \log n-\rho(M-2) \log \log n \\
& +O(\log \log \log n)\} \geq 1-O\left(\frac{1}{\log n}\right) .
\end{aligned}
$$

In particular

$$
\begin{aligned}
& \operatorname{Pr}\left\{\left|\frac{\max _{1 \leq i \leq n} \operatorname{SINR}_{i, m}}{\rho \log n}-1\right| \leq O\left(\frac{\log \log n}{\log n}\right)\right\} \\
& \geq 1-O\left(\frac{1}{\log n}\right) .
\end{aligned}
$$

Remark 1: Lemma 4 shows that when $M$ is fixed and $n$ increases, the maximum SINR behaves like

$$
\rho \log n+O(\log \log n) \text {. }
$$

On the other hand, from the expression for the SINR defined in (5), it is clear that the numerator is a $\chi^{2}(2)$ random variable and the interference terms constitute a $\chi^{2}(2 M-2)$ random variable. It is well known that (see Example 1 in Appendix A) the maximum of $n$ i.i.d. $\chi^{2}(2)$ behaves like $\log n$ for large $n$. One may then suspect that $\max _{i=1, \ldots, n} \mathrm{SINR}_{i, m}$ should behave like $\frac{\log n}{\frac{1}{2}+M-1}$, arguing that when the numerator takes on its maximum the denominator takes on its average value. What is interesting about Lemma 4 is that this heuristic argument is not true. It turns out that $\max _{i=1, \ldots, n} \operatorname{SINR}_{i, m}$ is achieved when the numerator behaves as $\log n$ and the interference terms are arbitrarily small, this yielding the behavior $\rho \log n$.

Proof: We use Corollary A.1 in Appendix A to find the asymptotic distribution of the maximum of $n$ i.i.d. random variable $\operatorname{SINR}_{i, m}$ for $i=1, \ldots, n$. The growth function $g_{s}(x)$ for $x \geq 0$ here is

$$
\begin{aligned}
g_{s}(x) & =\frac{1-F_{s}(x)}{f_{s}(x)}=\frac{\frac{e^{-x \rho}}{(1+x)^{M-1}}}{\frac{e^{-x / \rho}}{(1+x)^{M}}\left(\frac{1}{\rho}(1+x)+M-1\right)} \\
& =\frac{1+x}{(1+x) / \rho+M-1} \\
& =\rho-\frac{\rho(M-1)}{(1+x) / \rho+M-1} .
\end{aligned}
$$

Clearly, $\lim _{x \rightarrow \infty} g_{s}(x)=\rho>0$ so that the first condition of Corollary A. 1 is met. To verify the second condition, we need to find $u_{n}$ defined via the equation $1-F_{s}\left(u_{n}\right)=1 / n$. Thus,

$$
\begin{aligned}
1-F_{s}\left(u_{n}\right)= & \frac{e^{-u_{n} / \rho}}{\left(1+u_{n}\right)^{M-1}}=\frac{1}{n} \\
& \Longrightarrow \frac{u_{n}}{\rho}+(M-1) \log \left(1+u_{n}\right)=\log n .
\end{aligned}
$$

Equation (21) implies that

$$
u_{n}=\rho \log n-\rho(M-1) \log \log n+O(\log \log \log n)
$$

for large $n$ and fixed $M$. Taking derivatives, it is straightforward to verify that

$$
g^{(m)}\left(u_{n}\right)=O\left(\frac{1}{\left(1+u_{n}\right)^{m+1}}\right)
$$

for large $n$. Corollary A.1 therefore applies and so

$$
\begin{array}{r}
\operatorname{Pr}\left\{\rho \log \log n \leq \max _{1 \leq i \leq n} \operatorname{SINR}_{i, m}-u_{n} \leq \rho \log \log n\right\} \\
\geq 1-O\left(\frac{1}{\log n}\right) .
\end{array}
$$

The theorem follows by substituting the value of $u_{n}$ in (22).

We can now state the following theorem to prove the asymptotic linear scaling of the throughput with $M$ when $M$ is fixed.

Theorem 1: Let $M$ and $\rho$ be fixed and $N=1$. Then

$$
\lim _{n \rightarrow \infty} \frac{R}{M \log \log n}=1 .
$$

Proof: We derive upper and lower bounds for $R$ when $n$ is sufficiently large. For large $n$, using the upper bound in (8) and (19), we may write

$$
\begin{aligned}
R \leq & E\left\{\sum_{m=1}^{M} \log \left(1+\max _{i=1, \ldots, n} \mathrm{SINR}_{i, m}\right)\right\} \\
\leq & M \operatorname{Pr}\left\{\max _{i=1, \ldots, n} \mathrm{SINR}_{i, m} \leq u_{n}+\rho \log \log n\right\} \\
& \times \log \left(1+u_{n}+\rho \log \log n\right) \\
& +M \operatorname{Pr}\left\{\max _{i=1, \ldots, n} \mathrm{SINR}_{i, m} \geq u_{n}+\rho \log \log n\right\} \\
& \times \log (1+\rho n) \\
\leq & M \log \left(1+u_{n}+\rho \log \log n\right) \\
& +M O(1 / \log n) \log (1+\rho n) \\
\leq & M \log \left(1+u_{n}+\rho \log \log n\right)+O(1)
\end{aligned}
$$

where

$$
u_{n}=\rho \log n-\rho(M-1) \log \log n+O(\log \log \log n)
$$

as derived in Lemma 4 and we used the fact that the throughput is bounded by $M \log (1+\rho n)$ (the capacity of a MIMO point to point system with $M$ transmit and $n$ receive antennas). In order to find a lower bound, We can use the lower bound in (9) and Lemma 4 to write (24) at the top of the following page, where we used the definition of the conditional probability. The corollary follows by substituting the value of $u_{n}$ and observing that both the lower and upper bounds converge to $M \log \log n+$ $O(\log \log \log n)$. 


$$
\begin{aligned}
R & \geq \operatorname{Pr}\left\{\max _{i=1, \ldots, n} \operatorname{SINR}_{i, m} \geq 1\right\} E\left\{\sum_{m=1}^{M} \log \left(1+\max _{i=1, \ldots, n} \operatorname{SINR}_{i, m}\right) \mid \max _{i=1, \ldots, n} \operatorname{SINR}_{i, m} \geq 1\right\} \\
& \geq \operatorname{Pr}\left\{\max _{i=1, \ldots, n} \operatorname{SINR}_{i, m} \geq 1\right\} \times \frac{M \log \left(1+u_{n}-\rho \log \log n\right) \operatorname{Pr}\left\{\max _{i=1, \ldots, n} \operatorname{SINR}_{i, m} \geq u_{n}-\rho \log \log n\right\}}{\operatorname{Pr}\left\{\max _{i=1, \ldots, n} \operatorname{SINR}_{i, m} \geq 1\right\}} \\
& =M \log \left(u_{n}-\rho \log \log n\right)(1-O(1 / \log n))
\end{aligned}
$$

Remark 2: Using (18) it is not hard to obtain the next order term in $R$ as follows:

$$
R \geq M \log (\rho \log n-\rho M \log \log n)+o(\log \log \log n) .
$$

Theorem 1 states that for fixed $M$ as $n$ grows to infinity, the throughput scales like $M \log \log n$. Interestingly, in Lemma 1, we showed that $M \log \log n$ is in fact the best sum rate capacity that can be achieved with full knowledge of the channel using dirty paper coding [6]-[8]. Therefore, as far as the scaling law of the throughput is concerned, we are not losing anything in terms of the throughput provided that $M$ is fixed. This in fact raises the question of how far can we increase $M$ and still maintain the linearly scaling of throughput with $M$. This question will be answered in Section V.

\section{How Fast Can $M$ Grow to Retain LinEar Scaling OF ThroughPUT With $M$ ?}

In this section, we consider the case where the number of transmit antennas $M$ is allowed to grow to infinity. Similar to the previous section, we assume each receiver has a single antenna and the total average transmit power is $M$, i.e., the average transmit power per antennas is one.

Since $M$ is also going to infinity, the results in Appendix A do not apply. Therefore, we need to directly analyze the asymptotic behavior of the maximum SINR when both $n$ and $M$ grow to infinity. Of course, the asymptotics will depend on the growth rate of $M$ relative to $n$.

In what follows, we first show that if $\lim _{n \rightarrow \infty} \frac{M}{\log n}$ is a constant, the throughput of our scheme still exhibits linear growth, i.e., $\lim _{n \rightarrow \infty} \frac{R}{M}$ is a constant independent of $n$. Furthermore, we show that if $M$ grows faster than $\log n$, i.e., $\lim _{n \rightarrow \infty} \frac{M}{\log n}=\infty$, then the ratio of the throughput to $M$ tends to zero. Therefore, throughput will linearly scale with $M$ provided that $M$ does not grow faster than $\log n$.

Theorem 2: Suppose the transmitter has $M$ antennas, each receiver is equipped with a single antenna, and that we use random beamforming to users with the highest SINRs. Then, if $M=\frac{\log n+3 \log \log n-c / \rho}{\log (1+c)}+1$, where $c$ is a positive constant. Then

$$
\begin{aligned}
\operatorname{Pr}\left\{c-\alpha \frac{\log \log n}{\log n} \leq \max _{1 \leq i \leq n} \operatorname{SINR}_{i, m} \leq c\right\} & \\
\geq & 1-O\left(\frac{1}{\log ^{3} n}\right)
\end{aligned}
$$

where $\alpha=7(1+c) \log (1+c)$. Consequently

$$
\lim _{n \rightarrow \infty} \frac{R}{M \log (1+c)}=1 \text {. }
$$

Proof: First of all, note that

$$
1-F_{s}(c)=\frac{e^{-c / \rho}}{(1+c)^{M-1}}=e^{-c / \rho+(M-1) \log (1+c)} .
$$

Inserting the value of $M$ in (28) yields $1-F_{s}(c)=\frac{1}{n \log ^{3} n}$. Therefore,

$$
\begin{aligned}
\operatorname{Pr}\left\{\max _{1 \leq i \leq n} \operatorname{SINR}_{i, m} \leq c\right\}=\left\{F_{s}(c)\right\}^{n} & =\left(1-\frac{1}{n \log ^{3} n}\right)^{n} \\
& =e^{n \log \left(1-\frac{1}{n \log ^{3} n}\right)} \\
& =e^{-1 / \log ^{3} n+O\left(\frac{1}{n \log ^{6} n}\right)} \\
& =1-O\left(1 / \log ^{3} n\right)
\end{aligned}
$$

where we used the fact that $\log (1-x)=-x+O\left(x^{2}\right)$ for small $x$. Similarly setting

$$
c^{\prime}=c-\frac{\alpha \log \log n}{\log n}
$$

we have

$$
\begin{aligned}
1- & F_{s}\left(c^{\prime}\right) \\
= & e^{-c / \rho+\frac{\alpha \log \log n}{\rho \log n}+(M-1) \log (1+c)-(M-1) \log \left(1-\frac{\alpha \log \log n}{(1+c) \log n}\right)} \\
= & e^{-c / \rho+(M-1) \log (1+c)} \times e^{\frac{\alpha \log \log n}{\rho \log n}} \\
& \times e^{-(M-1) \log \left(1-\frac{\alpha \log \log n}{(1+c) \log n}\right)} \\
= & \frac{e^{\frac{\alpha \log \log n}{(1+c) \log (1+c)}+O(\log \log n / \log n)}}{n \log ^{3} n} \\
= & \frac{\log ^{4} n \times(1+O(\log \log n / \log n))}{n} \\
= & \frac{\log ^{4} n+o\left(\log ^{4} n\right)}{n}
\end{aligned}
$$

where in the third step we used $\alpha=7(1+c) \log (1+c)$ and in the forth step we used the identity $e^{x}=1+O(x)$ for small $x$. We can now state that

$$
\begin{gathered}
\operatorname{Pr}\left\{\max _{1 \leq i \leq n} \operatorname{SINR}_{i, m} \leq c-\frac{\alpha \log \log n}{\log n}\right\} \\
=\left(F_{s}\left(c^{\prime}\right)\right)^{n}=\left(1-\frac{\log ^{4} n+o\left(\log ^{4} n\right)}{n}\right)^{n} \\
=e^{-\log ^{4} n+o\left(\log ^{4} n\right)}=O\left(\frac{1}{n^{4}}\right)
\end{gathered}
$$


where in the last step we have been very conservative. Now, using (30) and (29), we get

$$
\begin{aligned}
\operatorname{Pr} & \left\{c-\alpha \frac{\log \log n}{\log n} \leq \max _{1 \leq i \leq n} \operatorname{SINR}_{i, m} \leq c\right\} \\
\geq & \operatorname{Pr}\left\{\max _{1 \leq i \leq n} \operatorname{SINR}_{i, m} \leq c\right\} \\
& +\operatorname{Pr}\left\{c-\alpha \frac{\log \log n}{\log n} \leq \max _{1 \leq i \leq n} \operatorname{SINR}_{i, m}\right\}-1 \\
= & 1-O\left(\frac{1}{\log ^{3} n}\right) .
\end{aligned}
$$

In order to find bounds on the throughput, we use a similar argument as in the proof of Theorem 1 to show (32) at the bottom of the page, where we used the fact the sum rate is bounded by $M \log (1+\rho n)$ and $M=O(\log n)$. In order to derive a lower bound for the throughput, for $c>1$ we can use Lemma 2 and the fact that the maximum SINR is almost surely equal to $c>1$, to obtain a lower bound as

$$
R \geq M \log \left(1+c-\alpha \frac{\log \log n}{\log n}\right)\left(1-O\left(\frac{1}{n^{4}}\right)\right)
$$

for $c>1$. Clearly, for $c<1$, the lower bound in Lemma 2 is not tight. Therefore, in order to find a lower bound, we define the event $A$ as the event that for all $m$

$$
c-\epsilon \leq \max _{1 \leq i \leq n} \operatorname{SINR}_{i, m} \leq c
$$

where $\epsilon=\alpha \frac{\log \log n}{\log n}$. We also define the event $B$ as the event that each user $i$ can at most be the maximum for one signal $s_{m}$.

Therefore, the throughput can be written as

$$
\begin{aligned}
R= & E\{\text { rate }\}=E\{\text { rate } \mid A \cap B\} \operatorname{Pr}\{A \cap B\} \\
& +E\left\{\text { rate } \mid A^{\prime} \cup B^{\prime}\right\}(1-\operatorname{Pr}\{A \cap B\}) \\
\geq & E\{\text { rate } \mid A \cap B\} \operatorname{Pr}\{A \cap B\} \\
\geq & M \log \left(1+c-\alpha \frac{\log \log n}{\log n}\right) \operatorname{Pr}\{B \mid A\} \operatorname{Pr}\{A\}
\end{aligned}
$$

where in the last inequality we used the fact that given the events $A$ and $B$, the transmission rate corresponding to the signal $s_{m}$ is greater than

$$
\log \left(1+c-\alpha \frac{\log \log n}{\log n}\right) .
$$

Now we can use the union bound to find a lower bound for $\operatorname{Pr}\{A\}$ as

$$
\begin{aligned}
\operatorname{Pr}\{A\} & =\operatorname{Pr}\left\{\forall m, \max _{1 \leq i \leq n} \operatorname{SINR}_{i, m} \in[c-\epsilon, c]\right\} \\
& \geq 1-\operatorname{Pr}\left\{\exists m, \max _{1 \leq i \leq n} \operatorname{SINR}_{i, m} \notin[c-\epsilon, c]\right\} \\
& \geq 1-M \operatorname{Pr}\left\{\max _{1 \leq i \leq n} \operatorname{SINR}_{i, m} \notin[c-\epsilon, c]\right\} \\
& =1-O\left(\frac{M}{\log ^{3} n}\right)
\end{aligned}
$$

where we used (31) in the last inequality. In Appendix C, it is shown that

$$
\operatorname{Pr}\{B \mid A\} \geq 1-O\left(\frac{(\log \log n)^{2}}{\sqrt{\log n}}\right) .
$$

Therefore, inserting (36) and (35) into (34), we get

$$
\begin{aligned}
R \geq M \log \left(1+c-\alpha \frac{\log \log n}{\log n}\right) & \left(1-O\left(\frac{(\log \log n)^{2}}{\sqrt{\log n}}\right)\right) \\
\times & \left(1-O\left(\frac{\log n}{\log ^{3} n}\right)\right) .
\end{aligned}
$$

Theorem 2 follows using (37) and (33) for $c \leq 1$.

Theorem 2 shows that when $M$ grows like $\log n$, the throughput still scales linearly with $M$. In the next theorem, we show that increasing $M$ at a rate faster than $\log n$ results in sublinear scaling of the throughput with $M$. It is also worth noting that the sum rate capacity of the broadcast channel with full CSI also scales linearly with $M$, i.e., $\lim _{n \rightarrow \infty} \frac{R^{D P}}{M}=\alpha$ where $\alpha$ is a constant independent of $n$ [14]. Therefore, in this regime, up to a constant multiplicative factor, the scaling law of the throughput of our scheme is still the same as that of dirty paper coding.

Remark 3: Similar to Remark 1, from the SINR expression one may expect that $\max _{i=1, \ldots, n} \operatorname{SINR}_{i, m}$ behaves as

$$
\frac{\log n}{M}=\frac{\log n}{\log n / \log (1+c)}=\log (1+c) .
$$

The argument being that the maximum is achieved when the numerator behaves as $\log n$ and the denominator behaves as the mean of $\chi^{2}(2 M-2)$ (here it is not reasonable to assume that the numerator can be $\log n$ and the interference terms arbitrarily

$$
\begin{aligned}
R \leq & E\left\{\sum_{m=1}^{M} \log \left(1+\max _{i=1, \ldots, n} \operatorname{SINR}_{i, m}\right)\right\} \\
= & \operatorname{Pr}\left\{\max _{i=1, \ldots, n} \operatorname{SINR}_{i, m} \leq c\right\} E\left\{\left.\sum_{m=1}^{M} \log \left(1+\max _{i=1, \ldots, n} \operatorname{SINR}_{i, m}\right)\right|_{i=1, \ldots, n} \max _{i, m} \operatorname{SINR}_{i, m} \leq c\right\} \\
& +\operatorname{Pr}\left\{\max _{i=1, \ldots, n} \operatorname{SINR}_{i, m} \geq c\right\} E\left\{\left.\sum_{m=1}^{M} \log \left(1+\max _{i=1, \ldots, n} \operatorname{SINR}_{i, m}\right)\right|_{i=1, \ldots, n} \max _{i, m} \geq c\right\} \\
\leq & M \log (1+c)+O\left(M \log n / \log ^{3} n\right) \\
= & M \log (1+c)+O(1 / \log n)
\end{aligned}
$$


small, since we have $O(\log n)$ interference terms). However, careful analysis of Theorem 2 shows that this heuristic is false: $\max _{i=1, \ldots, n} \mathrm{SINR}_{i, m}$ is achieved when the numerator behaves as $\log n$ and the interference terms as

$$
\frac{\log n}{c}<\frac{\log n}{\log (1+c)}
$$

Theorem 3: Consider the setting of Theorem 2. If $\lim _{n \rightarrow \infty} \frac{M}{\log n}=\infty$, then $\lim _{n \rightarrow \infty} \frac{R}{M}=0$.

Proof: Let $u_{n}$ be a positive sequence such that $\lim _{n \rightarrow \infty} u_{n}=0$. For such a $u_{n}$, let

$$
M=\frac{2 \log n}{\log \left(1+u_{n}\right)}+1 \text {; }
$$

clearly

$$
\lim _{n \rightarrow \infty} \frac{M}{\log n}=\infty
$$

if and only if $\lim _{n \rightarrow \infty} u_{n}=0$. With the choice of $u_{n}$, we have

$$
1-F_{s}\left(u_{n}\right)=e^{-u_{n} / \rho-(M-1) \log \left(1+u_{n}\right)}=\frac{e^{-u_{n} / \rho}}{n^{2}}
$$

and therefore,

$$
\begin{aligned}
\operatorname{Pr}\left\{\max _{1 \leq i \leq n} \operatorname{SINR}_{i, m} \leq u_{n}\right\}=\left\{F_{s}\left(u_{n}\right)\right\}^{n} & =\left(1-\frac{e^{-u_{n} / \rho}}{n^{2}}\right)^{n} \\
& \geq\left(1-\frac{1}{n^{2}}\right)^{n} \\
& =1-O\left(\frac{1}{n}\right) .
\end{aligned}
$$

Using a similar argument as in the proof of Theorem 2, we can therefore bound the throughput as

$$
R \leq M \log \left(1+u_{n}\right)+O\left(\frac{M \log n}{n}\right) .
$$

Equation (39) implies that $\lim _{n \rightarrow \infty} \frac{R}{M}=0$ since $\lim _{n \rightarrow \infty} u_{n}=0$.

It is worth mentioning that with full CSI at the transmitter, the sum rate capacity linearly scales with $M$ even when $M$ is of the order of $n$ [20]. This can be seen by a simple zero forcing beamforming scheme that creates $M$ parallel channels as long as the channel matrix is full rank [12]. Our scheme has access only to partial CSI, and can therefore only guarantee a linear scaling in $M$, provided that $M$ does not grow faster than $\log n$.

\section{ASYMPTOTIC ANALYSIS OF THE ThroughPUT: $N>1, M$ Is FIXED}

In the previous sections, we focused on the case where each receiver is equipped with only one antenna. When the users have multiple receive antennas, the sum-rate capacity of DPC (dirty paper coding) scales as $M \log \log n N$ [14]. In so far as our scheme is concerned, there are three distinct possibilities.

1) Treating each receive antenna as an independent user. In this case, we effectively have $n N$ single antenna receivers. Therefore, each receiver should feed back $N$ times the amount of information since each user has $N$ independent antennas and therefore it has $N$ maximum SINRs corresponding to each receive antenna. The transmitter then assigns $s_{m}$ for $m=1, \ldots, M$ to the antenna of that user with the highest SINR, i.e., $\max _{1 \leq i \leq n N} \operatorname{SINR}_{i, m}$. Since we have $n N$ i.i.d. SINRs, the maximization will be over $n N$ i.i.d. random variables instead of $n$ ones which was the case for $N=1$.

2) Assigning at most one beam to each user. In this case, the SINR can be written as

$$
\begin{aligned}
\operatorname{SINR}_{i, m}=\frac{\phi_{m}^{*} H_{i}^{*} H_{i} \phi_{m}}{1 / \rho+\sum_{k \neq m} \phi_{k}^{*} H_{i}^{*} H_{i} \phi_{k}} & \\
& m=1, \ldots, M
\end{aligned}
$$

where as in (1), $H_{i}$ is the $N \times M$ channel matrix for the $i$ th user. Again we send the symbol $s_{m}$ to the user corresponding to $\max _{1 \leq i \leq n} \mathrm{SINR}_{i, m}$. Note that in this case each user just feeds back its maximum SINR and the corresponding index $m$ in which it is maximum.

3) Assigning multiple beams to each user. For simplicity, let us assume $K=M / N$ is an integer. ${ }^{5}$ In this case, we either assign $N$ beams to a user or no beams at all. Therefore, to find the best user, instead of feeding back SINRs, each receiver has to feed back its capacity, computed as in (41) at the bottom of the page, where the $\Phi_{k}$ 's $(k=1, \ldots, K)$ are $M \times N$ random orthonormal matrices chosen according to an isotropic distributions. In other words, $\Phi=\left(\Phi_{1} \ldots \Phi_{K}\right)$ is an $M \times M$ unitary matrix.

As mentioned earlier, the first case is effectively the same as having $n N$ users with single receive antennas. The second case is a generalization of the case with $N=1$, and it turns out the analysis of this is very similar to that of the case with $N=1$. On the other hand, the last case is quite different from the previous two and requires more effort to be analyzed. In terms of the amount of feedback, clearly the first case requires $N$ times more feedback than that of the second and third cases.

Case 1:

Here, since we consider all the receive antennas as separate users (no cooperation among receivers), we have $n N$ users with single receive antennas. In this case, the formulation of the problem is the same as that in Section IV with the only difference being that $n$ is replaced by $n N$. Therefore, we can

\footnotetext{
${ }^{5}$ The more general case can be handled in a straightforward fashion, but will not be done here for the sake of brevity.
}

$$
C_{i, m}=\log \left\{\operatorname{det}\left\{I+\Phi_{m}^{*} H_{j}^{*} H_{j} \Phi_{m}\left(\frac{1}{\rho} I+\sum_{k \neq m} \Phi_{k}^{*} H_{j}^{*} H_{j} \Phi_{k}\right)^{-1}\right\}\right\}, \quad m=1, \ldots, M / N
$$


state the following limit result as a simple consequence of Theorem 1:

$$
\lim _{n \rightarrow \infty} \frac{R}{M \log \log n N}=1
$$

when $M$ is fixed and for any $N$.

Remark 4: In fact, it has been recently shown in [14] that when $M$ is fixed, $n$ is large, and for any $N$, the sum rate capacity scales like $M \log \log n N$ in the presence of full CSI in the transmitter using dirty paper coding. Therefore, treating each antenna as an independent user does give the right scaling law for the throughput.

Case 2:

Here we send at most one symbol per user. Therefore, each user has to feed back its maximum SINR calculated as in (40), where $H_{i}$ is the $N \times M$ channel matrix for the $i$ th user. Similar to Section IV, using the orthogonality of $\phi_{m}$ 's, we first write the $\operatorname{SINR}$ as $\operatorname{SINR}_{i, m}=\frac{z}{1 / \rho+y}$ where $z$ has $\chi^{2}(2 N)$ and $y$ has $\chi^{2}(2 N(M-1))$ distributions that are independent. ${ }^{6}$ Therefore, we may write the pdf of the $\operatorname{SINR}_{i, m}$ (denoted by $f_{s_{N}}$ ) as

$$
\begin{aligned}
f_{s_{N}}(x)= & \frac{x^{2 N-2} e^{-x / \rho}}{(2 N-1) !(N M-N-1) !} \\
& \times \int_{0}^{\infty}(u+1 / \rho)^{2 N-1} u^{N M-N-1} e^{-u(1+x)} d u \\
= & \frac{x^{2 N-2} e^{-x / \rho}}{(2 N-1) !(N M-N-1) !} \sum_{i=0}^{2 N-1}\left(\begin{array}{c}
2 N-1 \\
i
\end{array}\right) \\
& \times \frac{1}{\rho^{2 N-i-1}} \times \frac{(N(M-1)+i-1) !}{(1+x)^{N(M-1)+i}} .
\end{aligned}
$$

The preceding $f_{s_{N}}(x)$ can be used to evaluate exactly the throughput using Lemma 2. The asymptotic analysis can be done similarly to that of Section IV, although the analysis becomes more cumbersome.

Theorem 4: Let $\max _{1 \leq i \leq n} \operatorname{SINR}_{i, m}$ where $\operatorname{SINR}_{i, m}$ for $i=$ $1, \ldots, n$ be $n$ i.i.d. random variables defined in (40) and let $M$ and $N$ be fixed numbers. Then for sufficiently large $n$, we get (44) at the bottom of the page, and therefore,

$$
\lim _{n \rightarrow \infty} \frac{R}{M \log \log n}=1 \text {. }
$$

Proof: We use Corollary A.1 in Appendix A to prove the first part of the theorem. We first check whether the growth function has a positive constant limit or not. Using Hopital's rule and (43) we get

$$
\lim _{x \rightarrow \infty} g_{s_{N}}(x)=\lim _{x \rightarrow \infty} \frac{-f_{s_{N}}(x)}{f_{s_{N}}^{\prime}(x)}=\rho>0 .
$$

${ }^{6}$ The reason being that $\Phi$ is a unitary matrix and $H_{i}$ is a matrix of i.i.d. $C N(0,1)$.
So the first condition in Corollary A.1 is met. Furthermore, taking the integral of $f_{s_{N}}(x)$ in (43), it is quite straightforward to show that $g_{s_{N}}^{(m)}(x)=O\left(1 / x^{m}\right)$ for large $x$.

To verify the last condition, we need to find $u_{n}$ defined as the solution of $1-F_{s_{N}}\left(u_{n}\right)=\frac{1}{n}$. Since solving the equation is involved, we can find upper and lower bounds for $u_{n}$, i.e., $u_{n}^{l} \leq u_{n} \leq u_{n}^{u}$, by first deriving lower and upper bounds for $f_{s_{N}}(x)$

$$
\begin{aligned}
& \left(\begin{array}{c}
N M+N-2 \\
2 N-1
\end{array}\right) \frac{e^{-x / \rho}}{(1+x)^{N(M+1)-3}} \leq f_{s_{N}}(x) \\
& \leq \frac{\sum_{i=0}^{2 N-1}\left(\begin{array}{c}
2 N-1 \\
i
\end{array}\right) \frac{(N(M-1)+i-1) !}{\rho^{2 N-i-1}}}{(2 N-1) !(N M-N-1) !} \frac{e^{-x / \rho}}{(1+x)^{N(M-3)+2}}
\end{aligned}
$$

for $x>1$. The lower bound follows by replacing $u+1 / \rho$ by $u$ in the integral of (43) which then becomes an exponential integral. The upper bound can be also derived by using $x<x+1$ and $1<x+1$ in the expansion in (43).

Replacing $f_{s_{N}}(x)$ by its lower bound allows us to compute $u_{n}^{l}$ via

$$
\int_{u_{n}^{l}}^{\infty} \frac{\gamma_{1} e^{-x / \rho}}{(1+x)^{N(M+1)-3}} d x=\frac{1}{n}
$$

where $\gamma_{1}=\left(\begin{array}{c}N M+N-2 \\ 2 N-1\end{array}\right)$. Using the identity

$$
\int_{a}^{\infty} \frac{e^{-b x}}{(1+x)^{M}} d x=e^{b} b^{M-1} \Gamma(-M+1, b(a+1))
$$

and the asymptotic expansion

$$
\Gamma(-M+1, x)=x^{M} e^{-x}(1+O(1 / x))
$$

for large $|x|$ as in [21], we obtain

$$
\begin{aligned}
& \gamma_{1}^{\prime} \Gamma\left(-M+1, \frac{1}{\rho}\left(u_{n}^{l}+1\right)\right)=\frac{1}{n} \\
& \Longrightarrow u_{n}^{l}=\rho \log n-\rho(N(M+1)-3) \log \log n \\
&+O(\log \log \log n)
\end{aligned}
$$

where $\gamma_{1}^{\prime}$ only depends on $N, M, \rho$, and does not depend on $n$.

We can similarly find the upper bound as

$u_{n}^{u}=\rho \log n+\rho(N(M-3)+1) \log \log n+O(\log \log \log n)$.

Using Corollary A.1 and the bounds for $u_{n}$, we can use the same argument as in the proof of Theorem 1 (i.e., (24)), to prove the first part of the theorem. The second part of the theorem follows by using the same argument as in the proof of Theorem 1 (i.e., (24)) and (44).

Remark 5: Using (44) it follows that the next order term in $R$ is

$$
\begin{aligned}
R \leq M \log (\rho \log n-\rho(N(M-3)-1) \log \log n) \\
+o(\log \log \log n) .
\end{aligned}
$$

$$
\begin{aligned}
\operatorname{Pr}\{\rho \log n- & \rho(N(M+1)-2) \log \log n+O(\log \log \log n) \\
& \left.\leq \max _{1 \leq i \leq n} \operatorname{SINR}_{i, m}-O(\log \log \log n) \leq \rho \log n-\rho(N(M-3)+1) \log \log n \int x^{2} d x\right\} \geq 1-O\left(\frac{1}{\log n}\right)
\end{aligned}
$$


Note that using (25), the expansion for Case 1 is

$$
R \geq M \log (\rho \log n N-\rho M \log \log n N)+o(\log \log \log n)
$$

which implies that the scheme of Case 2 is worse than that of Case 1 (it is even worse than using $N=1$ receive antenna, which can be explained by the channel hardening that occurs for $N>1$ ).

\section{Case 3:}

Here each user feeds back its largest capacity $C_{i, m}$ defined in (41), and so we need to analyze the (asymptotic) distribution of $\max _{i=1 \ldots, n} C_{i, m}$ to find an upper bound for the throughput. While, in principle, this can be done, the algebra is extremely tedious. It turns out that an upper bound for the throughput can be derived if we replace $C_{i, m}$ by the simple upper bound

$$
C_{i, m} \leq C_{i, m}^{u}=\log \operatorname{det}\left(I+\rho \Phi_{m}^{*} H_{i}^{*} H_{i} \Phi_{m}\right) .
$$

The analysis of $C_{i, m}^{u}$ is easier because the eigenvalues of $\Phi_{m}^{*} H_{i}^{*} H_{i} \Phi_{m}$ are readily characterized (via Wishart distribution [22]) than those appearing in $C_{i, m}$. Since $H_{i} \Phi$ has the same distribution as $H_{i}$, the $N \times N$ matrices $H_{i} \Phi_{m}$ consists of i.i.d. $C N(0,1)$ and are also independent over $i$ and $m$. Therefore, we need to study

$$
C_{i}^{u}=\log \operatorname{det}\left(I+\rho G_{i}^{*} G_{i}\right), \quad i=1, \ldots, n
$$

where $G_{i}$ is $N \times N$ and has i.i.d. $C N(0,1)$ entries. Consequently, the throughput will be

$$
R \leq \frac{M}{N} E\left\{\max _{1 \leq i \leq n}\left(C_{1}^{u}, \ldots, C_{n}^{u}\right)\right\}
$$

since we have $M / N$ random beams. Now letting $\lambda_{1}^{i}, \ldots, \lambda_{N}^{i}$ be the eigenvalues of the matrix $G_{i}^{*} G_{i}$, we can state the following inequality for $R$ :

$$
R \leq M E\left\{\max _{1 \leq i \leq n} \log \left(1+\frac{\rho}{N} \sum_{k=1}^{N} \lambda_{k}^{i}\right)\right\}
$$

where we used the inequality

$$
\operatorname{det}\left(I+G_{i}^{*} G_{i}\right) \leq\left(1+\frac{\operatorname{tr}\left(G_{i}^{*} G_{i}\right)}{N}\right)^{N} .
$$

The next theorem presents an asymptotic result for the throughput of Case 3.

Theorem 5: Let $M$ and $N$ be fixed and $n$ increasing, then the throughput of Case 3 is bounded by

$$
R \leq M \log \left(1+\frac{\rho}{N} \log n+O(\log \log n)\right) .
$$

Proof: In order to evaluate the upper bound in (53), we may use the fact that $\operatorname{tr}\left\{G^{*} G\right\}$ has $\chi^{2}\left(2 N^{2}\right)$ distribution. It is in fact shown in Example 1 of Appendix A that for large $n$, the maximum of $n \chi^{2}\left(2 N^{2}\right)$ random variables satisfies the equation at the bottom of the page. Therefore, we can use the preceding result, (53), and using the same argument as in Theorem 1 (i.e., (24)) to show that

$$
R \leq M \log \left(1+\frac{\rho}{N} \log n+O(\log \log n)\right)+O(1) .
$$

Again when $M$ and $N$ are fixed, the throughput achieved by Case 3 has the leading order term of $M \log \log n$. The only effect is observed in the lower order terms (the $\log \log \log n$ term), and therefore, we conclude that Case 1 is the best and Case 3 is the worst.

\section{A. Discussion}

The analysis of the sum rate capacity of DPC shows that increasing the number of receive antennas beyond $N=1$ does not substantially increase the total throughput [14]. Therefore, one may ask whether it is beneficial for any user to have more than one antenna. Thus, assume that some users have $N>1$ antennas, and that we are employing the scheme of Case 1. It is quite clear that a user with $N$ antennas will receive $N$ times the rate of a user with one antenna simply because the probability that it will be the strongest user and be transmitted to increases $N$-fold. Thus, users with more antennas will receive higher rates. However, since more receive antennas does not increase the throughput, this will come at the expense of all users in the system.

\section{FAIRNESS IN SCHEDULING}

So far, we have assumed a homogeneous network in the sense that the SNR for all users was equal, namely, $\rho=\rho_{i}, i=$ $1, \ldots, n$. In practice, however, due to the different distances of the users from the base station and the corresponding different path losses, the users will experience different SNRs so that $\rho_{i}$ 's will not be identical. Such networks are called heterogeneous.

In heterogeneous networks, there is usually tension between the gains obtained from employing multiuser diversity and the fairness of the system. More explicitly, if we transmit only to the best user to maximize the throughput, the system may be dominated by users that are closest to the base station. On the other hand, if we insist on transmitting to users in a fair way (for example, by insisting on proportional fairness [13]), then we will be sacrificing throughput since we will not always be transmitting to the strongest user.

A fortunate consequence of our random multibeam method is that, if the number of transmit antennas is large enough then the system becomes interference dominated. In other words, even though the closest users will receive strong signal, they will also receive strong interference. In this case, being the best user will depend not so much on how close one is to the base station, but rather on how one's channel vector $H_{i}$ aligns with the closest

$$
\operatorname{Pr}\left\{\log n+\left(N^{2}-2\right) \log \log n+O(\log \log \log n) \leq \max _{1 \leq i \leq n} \sum_{k=1}^{N} \lambda_{k}^{i}<\log n+N^{2} \log \log n+O(\log \log \log n)\right\} \geq 1-O(1 / \log n)
$$


beam direction $\phi_{m}, m=1, \ldots, M$. Therefore, one would expect that the probability that any user is the strongest will not depend on its SNR $\rho_{i}$.

In what follows, we will make this observation more precise. We will show that if the number of transmit antennas $M$ grows faster than or equal to $\log n$ then the system will be fair, thus we achieve maximum throughput and fairness simultaneously.

As usual, we consider $M$ transmit antennas and $N=1$ receive antennas at each user (recall that for $N>1$, the best policy is to have no cooperation between $N$ antennas which basically changes the problem to $n N$ single-antenna users). Denoting the SNR of the $i$ th user by $\rho_{i}$, then the pdf of $\operatorname{SINR}_{i, m}$ can be written as

$$
f_{s, i}(x)=\frac{1 / \rho_{i} e^{-x / \rho_{i}}}{(1+x)^{M-1}}+\frac{(M-1) e^{-x / \rho_{i}}}{(1+x)^{M}}, \quad x \geq 0 .
$$

We are interested in computing the probability of transmitting the $m$ th signal to the $i$ th user with SNR of $\rho_{i}$ (denoted by $P_{\rho_{i}, m}$ ), i.e.,

$$
\begin{aligned}
P_{\rho_{i}}= & P_{\rho_{i}, m}=\operatorname{Pr}\left\{\operatorname{SINR}_{i, m}>\operatorname{SINR}_{1, m}, \ldots,\right. \\
= & \int_{0}^{\infty} \int_{0}^{x_{i}} \cdots \int_{0}^{x_{i}} f_{\rho_{i}}\left(x_{i}\right) \\
& \times \prod_{j=1, j \neq i}^{n} f_{\rho_{j}}\left(x_{j}\right) d x_{1}, \ldots, d x_{n} .
\end{aligned}
$$

Note that due to the fact that $\operatorname{SINR}_{i, m}$ for $m=1, \ldots, M$ have identical distribution, $P_{\rho_{i}, m}$ does not depend on the index $m$ and $P_{\rho_{i}}=P_{\rho_{i}, m}$ for $m=1, \ldots, M$. The following theorem obtains bounds on the probability of choosing the weakest user with $\rho_{\min }=\min _{1 \leq i \leq n} \rho_{i}$ and the strongest user with $\rho_{\max }=$ $\max _{1 \leq i \leq n} \rho_{i}$.

Theorem 6: Let $M$ be the number of transmit antennas and $\rho_{i}$ is the SINR of the $i$ th user. Define

$$
\rho_{\min }=\min _{1 \leq i \leq n} \rho_{i} \quad \text { and } \quad \rho_{\max }=\max _{1 \leq i \leq n} \rho_{i}
$$

the SNR corresponding to the weakest and strongest user, respectively. Then

$$
P_{\rho_{\min }} \geq \frac{M-1+\frac{1}{\rho_{\min }}}{M-1+\frac{1}{\rho_{\max }}} \frac{e^{-\left(\frac{1}{\rho_{\min }}-\frac{1}{\rho_{\max }}\right)\left(e^{\frac{2 \log n}{M-1}}-1\right)}}{n}+O\left(\frac{1}{n^{2}}\right)
$$

and

$$
P_{\rho_{\max }} \leq \frac{e^{\left(\frac{1}{\rho_{\min }}-\frac{1}{\rho_{\max }}\right)\left(e^{\frac{2 \log n}{M-1}}-1\right)}}{n}+\frac{1}{n^{2}}
$$

where $P_{\rho_{\min }}$ and $P_{\rho_{\max }}$ are the probability of choosing users with minimum and maximum SNR, respectively.

Proof: Let

$$
\epsilon=\frac{1}{\rho_{\min }}-\frac{1}{\rho_{\max }} \geq 0 .
$$

We first find a lower bound for the probability of choosing the user with minimum SNR by assuming all the other users have the maximum SNR. Therefore, using (57), we get (60) at the bottom of the page. Also, we can use the following inequality for $x>0$ :

$$
\begin{aligned}
\frac{M-1+\frac{1}{\rho_{\max }}+\epsilon}{M-1+\frac{1}{\rho_{\max }}} & \leq \frac{M-1+\left(\frac{1}{\rho_{\max }}+\epsilon\right)(1+x)}{M-1+\frac{1+x}{\rho_{\max }}} \\
& \leq \frac{\frac{1}{\rho_{\max }}+\epsilon}{\frac{1}{\rho_{\max }}}
\end{aligned}
$$

where we used the fact that the function being bounded is monotonically increasing for $x \geq 0$. Now we define $u_{0}$ to be the solution to

$$
\frac{e^{-\frac{u_{0}}{\rho_{\max }}}}{\left(1+u_{0}\right)^{M-1}}=\frac{1}{n^{2}}
$$

Clearly, $\frac{e^{-\frac{x}{\rho \max }}}{(1+x)^{M-1}}$ is monotonically decreasing and there is a unique solution for $u_{0}$. Then for $\epsilon>0$, we get the second equation at the bottom of the page. In order to find a lower bound, we ignore the second integral and we use the fact that $e^{-\epsilon x}>e^{-\epsilon u_{0}}$ for $\epsilon>0$ and $0 \leq c \leq u_{0}$. Therefore, we get

$$
\begin{aligned}
P_{\rho_{\min }} \geq & \frac{M-1+\frac{1}{\rho_{\max }}+\epsilon}{M-1+\frac{1}{\rho_{\max }}} \\
& \times\left\{\frac{e^{-\epsilon u_{0}}}{n}\left(1-\frac{e^{-\frac{u_{0}}{\rho_{\max }}}}{\left(1+u_{0}\right)^{M-1}}\right)^{n}\right\} \\
= & \frac{M-1+\frac{1}{\rho_{\max }}+\epsilon}{M-1+\frac{1}{\rho_{\max }}} \frac{e^{-\epsilon u_{0}^{u}}}{n}\left(1-1 / n^{2}\right)^{n-1}
\end{aligned}
$$

$$
\begin{aligned}
P_{\rho_{\min }} & \geq \int_{0}^{\infty} \frac{e^{-\left(\frac{1}{\rho_{\max }}+\epsilon\right) x}}{(1+x)^{M}}\left\{\left(\frac{1}{\rho_{\max }}+\epsilon\right)(1+x)+M-1\right\}\left(1-\frac{e^{-\frac{x}{\rho_{\max }}}}{(1+x)^{M-1}}\right)^{n-1} d x \\
& \geq \int_{0}^{\infty} e^{-\epsilon x} \frac{M-1+\left(\frac{1}{\rho_{\max }}+\epsilon\right)(1+x)}{M-1+\frac{(1+x)}{\rho_{\max }}}\left(1-\frac{e^{-\frac{x}{\rho_{\max }}}}{(1+x)^{M-1}}\right)^{n-1} \frac{d}{d x}\left(-\frac{e^{-\frac{x}{\rho_{\max }}}}{(1+x)^{M-1}}\right) d x .
\end{aligned}
$$

$$
\begin{aligned}
P_{\rho_{\min }} \geq \frac{M-1+\frac{1}{\rho_{\max }}+\epsilon}{M-1+\frac{1}{\rho_{\max }}}\left\{e^{-\epsilon u_{0}} \int_{0}^{u_{0}}\left(1-\frac{e^{-\frac{x}{\rho_{\max }}}}{(1+x)^{M-1}}\right)^{n-1} \frac{d}{d x}\left(-\frac{e^{-\frac{x}{\rho_{\max }}}}{(1+x)^{M-1}}\right) d x\right. \\
\left.\quad+e^{-\epsilon u_{0}} \int_{u_{0}}^{\infty}\left(1-\frac{e^{-\frac{x}{\rho_{\max }}}}{(1+x)^{M-1}}\right)^{n-1} \frac{d}{d x}\left(-\frac{e^{-\frac{x}{\rho_{\max }}}}{(1+x)^{M-1}}\right) d x\right\} .
\end{aligned}
$$




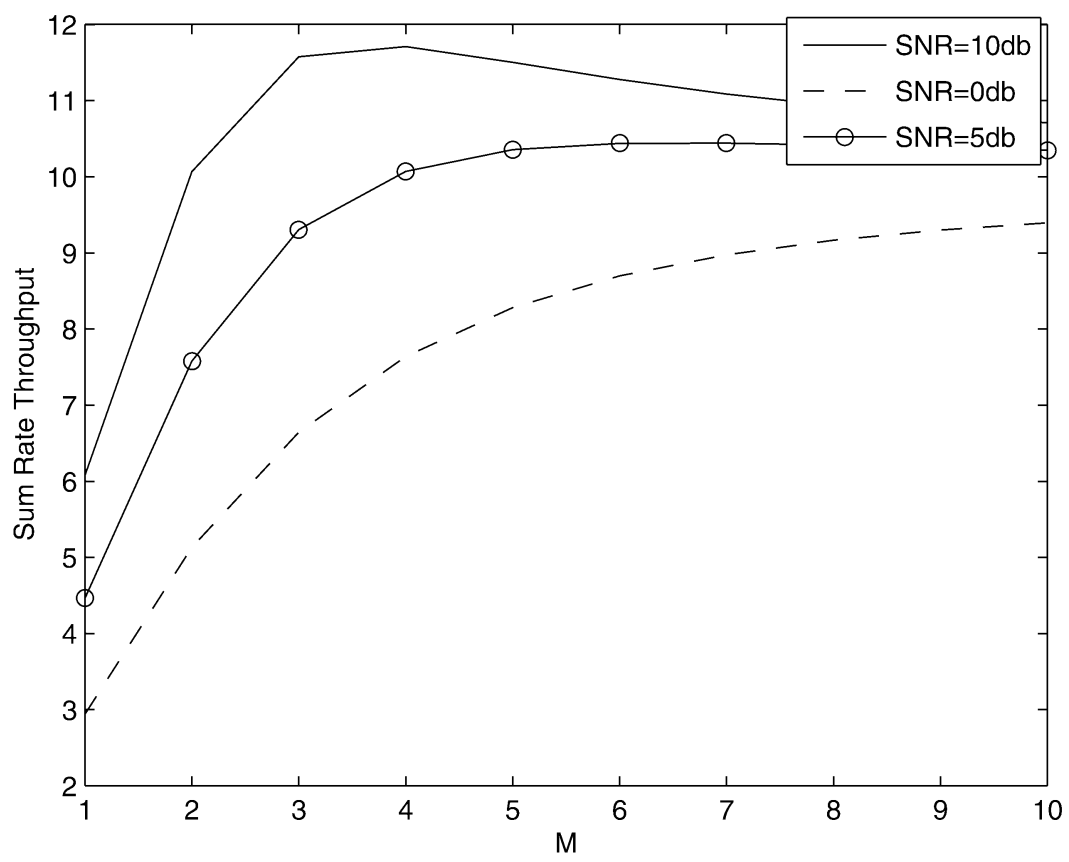

Fig. 1. Throughput versus the number of transmit antennas for different SNRs and $n=500$.

where $u_{0}^{u}$ is an upper bound for $u_{0}$, i.e., $u_{0}^{u}>u_{0}$, and can be calculated as

$$
\begin{aligned}
\frac{1}{\rho_{\max }} u_{0}+(M-1) \log \left(1+u_{0}\right) & =2 \log n \Longrightarrow u_{0} \leq n^{\frac{2}{M-1}}-1 \\
& =e^{\frac{2 \log n}{M-1}}-1=u_{0}^{u} .
\end{aligned}
$$

Therefore, for any $M$ and $n$, the lower bound can be written as

$$
\begin{aligned}
P_{\rho_{\text {min }}} \geq & \frac{M-1+\frac{1}{\rho_{\min }}}{M-1+\frac{1}{\rho_{\max }}} \\
& \times \frac{e^{-\left(\frac{1}{\rho_{\min }}-\frac{1}{\rho_{\max }}\right)\left(e^{\frac{2 \log n}{M-1}}-1\right)}}{n}\left(1-1 / n^{2}\right)^{n-1}
\end{aligned}
$$

which leads to (58). We can also find an upper bound for $P_{\rho_{\max }}$ by considering that all the other receivers have the minimum SNR. Therefore, similar to (60), we may write

$$
\begin{aligned}
P_{\rho_{\max }} \leq \int_{0}^{\infty} \frac{e^{-\frac{x}{\rho_{\max }}}}{(1+x)^{M}} & \left\{\frac{(1+x)}{\rho_{\max }}+M-1\right\} \\
& \times\left(1-\frac{e^{-\left(\frac{1}{\rho_{\max }}+\epsilon\right) x}}{(1+x)^{M-1}}\right)^{n-1} d x
\end{aligned}
$$

where we used the definition of $\epsilon$. We can further define

$$
h(x)=\frac{e^{-\frac{x}{\rho_{\max }}}}{(1+x)^{M-1}}
$$

and we let $u_{o}$ be the solution to $h\left(u_{0}\right)=1 / n^{2}$. Therefore, separating the integral to two regions, we get

$$
\begin{aligned}
P_{\rho_{\max }} \leq & \int_{0}^{u_{0}}\left(-h^{\prime}(x)\right)\left(1-h(x) e^{-\epsilon x}\right)^{n-1} d x \\
& +\int_{u_{0}}^{\infty}\left(-h^{\prime}(x)\right)\left(1-h(x) e^{-\epsilon x}\right)^{n-1} d x \\
\leq & \int_{0}^{u_{0}}\left(-h^{\prime}(x)\right)\left(1-h(x) e^{-\epsilon u_{0}}\right)^{n-1} d x \\
& +\int_{u_{0}}^{\infty}\left(-h^{\prime}(x)\right) d x
\end{aligned}
$$

where we used the fact that $1-h(x) e^{-\epsilon x}<1-h(x) e^{-\epsilon u_{0}}$ for $0 \leq x \leq u_{0}$ and $\epsilon>0$. Similarly, for the second integral we used $1-h(x) e^{-\epsilon x} \leq 1$. Noting that $h\left(u_{0}\right)=\frac{1}{n^{2}}$, the upper bound can be written as

$$
\begin{aligned}
P_{\rho_{\max }} & \leq \frac{e^{\epsilon u_{0}}}{n}\left\{\left(1-h\left(u_{0}\right) e^{-\epsilon u_{0}}\right)^{n}-\left(1-e^{-\epsilon u_{0}}\right)^{n}\right\}+h\left(u_{0}\right) \\
& \leq \frac{e^{\epsilon u_{0}}}{n}+\frac{1}{n^{2}}
\end{aligned}
$$

where $u_{0} \leq u_{0}^{u}$. We can therefore use the fact that $u_{0} \leq$ $e^{\frac{2 \log n}{M-1}}-1$ as shown in (62), to get

$$
P_{\rho_{\max }} \leq \frac{e^{\left(\frac{1}{\rho_{\min }}-\frac{1}{\rho_{\max }}\right)\left(e^{\frac{2 \log n}{M-1}}-1\right)}}{n}+\frac{1}{n^{2}}
$$

which leads to (59).

Based on the result of Theorem 6, we can state the following Corollary.

Corollary 1: If $\frac{M}{\log n}=\alpha$ then by increasing the average transmit power, we have $P_{\rho_{\min }} \rightarrow \frac{1}{n}$, and so the system becomes more and more fair. Alternatively, if we fix the SNR and increase $\alpha, P_{\rho_{\min }} \rightarrow \frac{1}{n}$ and the system becomes fair.

Proof: It is clear from Theorem 6 that if $\frac{M}{\log n}$ is fixed and we increase the average power, $P_{\rho_{\min }}$ is going to $\frac{1}{n}$. Moreover, as $\frac{M}{\log n}$ goes to infinity, again $P_{\rho_{\min }}$ is approaching $\frac{1}{n}$. Therefore, the system becomes fair.

\section{SIMULATION RESULTS}

In this section, we verify our asymptotic results with simulations and numerical evaluation. As Lemma 2 states, bounds on the throughput can be evaluated for any $n, M$, and $\rho$. We also proved in Theorems 1 and 2 that the upper bound is tight when $M \leq \alpha \log n$ which is the region that we are interested in, therefore, we plot (7) as a good approximation for the throughput. Figs. 1 and 2 show the throughput versus the number of transmit 


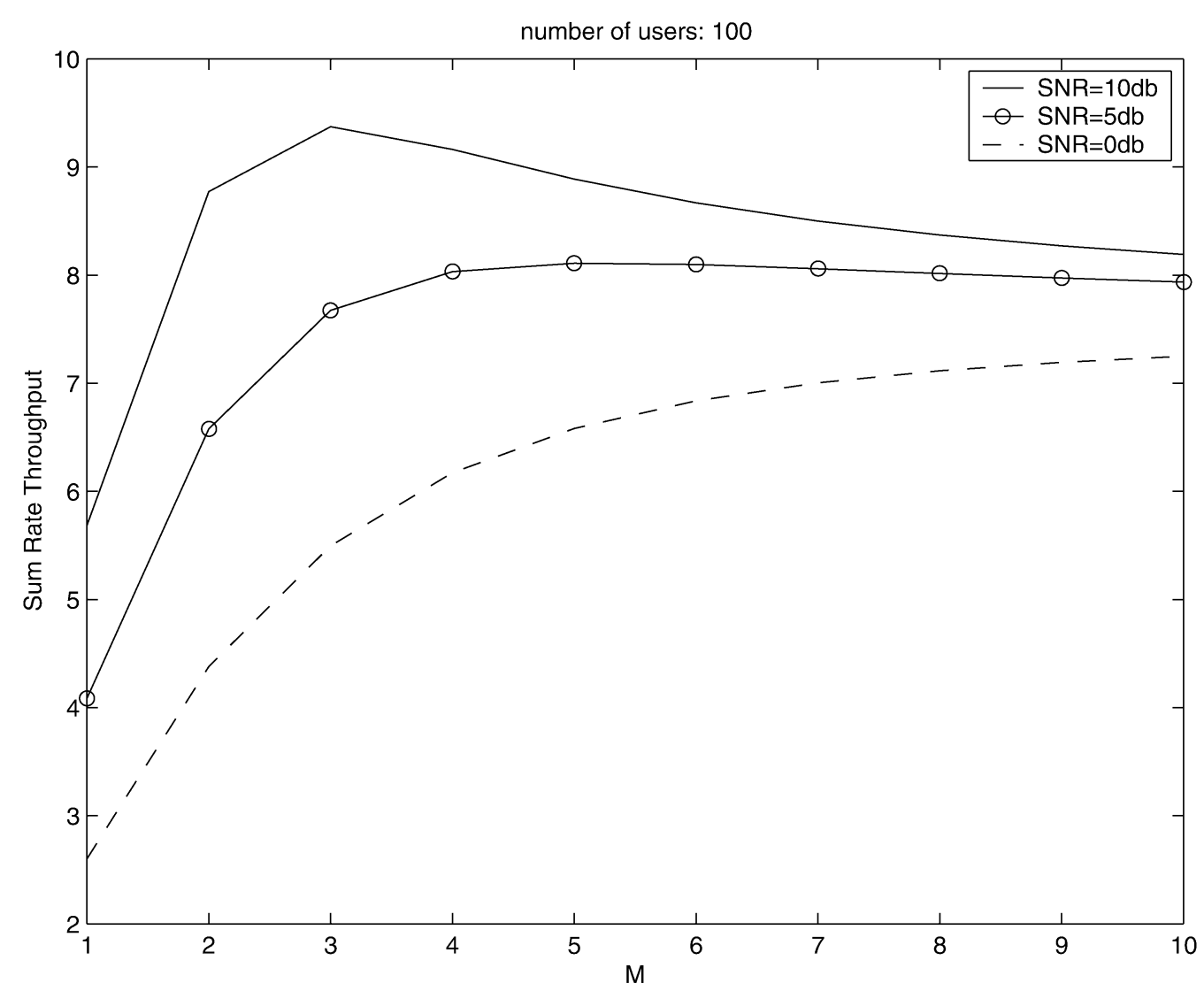

Fig. 2. Throughput versus the number of transmit antennas for different SNRs and $n=100$.

antennas $M$, for different SNRs. Clearly, for $M \leq 4$ the curve behaves linearly and as $M$ becomes $\log n \approx 4$ the throughput curves become saturated.

We also investigate the fairness of the scheduling by simulations. We compare the fairness of our scheduling with multiple transmit antennas with that of the case with one antenna in the base station $M=1$, in which the base scheduling strategy (in terms of maximizing the throughput) is to transmit to the user with the maximum SNR. Suppose users have SNRs uniformly distributed from 6 to $15 \mathrm{~dB}$, therefore, the users corresponding to the SNR of 15 and $6 \mathrm{~dB}$ are the strongest and the weakest users, respectively. Fig. 3 shows the number of times that each user with the corresponding SNR is chosen out of 50000 iterations. Clearly, the user with the minimum SNR rarely gets to be transmitted to. On the other hand, Fig. 4 shows the fairness of our proposed algorithm by using $M=5(\approx \log n)$ antennas in the base station. As Figs. 3 and 4 show, the fairness has been significantly improved by using multiple transmit antennas. For instance, the ratio of the number of times that the strongest user is chosen to the number of times that the weakest user is chosen, is 700 for the case with $M=1$ as opposed to 2.5 for the case with $M=5$ using our scheduling.

\section{CONCLUSION}

This paper deals with multiple-antenna broadcast channels where due to rapid time variations of the channel, limited resources, imperfect feedback, full CSI for all users cannot be provided at the transmitter. Since having no CSI does not lead to gains, it is important to study MIMO broadcast channels withpartial CSI. In this paper, we proposed using random beams and choosing the users with the highest signal-to-interference ratios. When the number of users grows and $M$ is fixed, we proved that the throughput scales like $M \log \log n$ which coincides with the scaling law of the sum-rate capacity assuming perfect CSI and using dirty paper coding. We further showed that with our scheme, the throughput scales linearly with $M$, provided that $M$ does not grow faster than $\log n$. Moreover, we considered different scenarios for the case with more than one receive antenna $N>1$, and we showed that by using random beamforming, the throughput of our scheme scales as $M \log \log n N$ when $M$ is fixed and for any $N$ which is precisely the same as the scaling of the sum rate capacity using dirty paper coding. This implies that increasing $N$ has no significant impact on the throughput.

Another issue that we addressed is to analyze the fairness in our scheduling when the users are heterogeneous. We proved that as $M$ becomes large, the scheduling becomes more and more fair and when $M>\alpha \log n$, the scheduling will be fair irrespective of the SNR of the users. We conclude that using $M=\alpha \log n$ emerges as a desirable operating point, both in terms of guaranteeing fairness as well as providing linear scaling of the throughput with $M$.

APPENDIX A

\section{ON EXTREME VALUE THEORY}

In this appendix, we review some results on the asymptotic behavior of the maximum of $n$ i.i.d. random variables when $n$ 


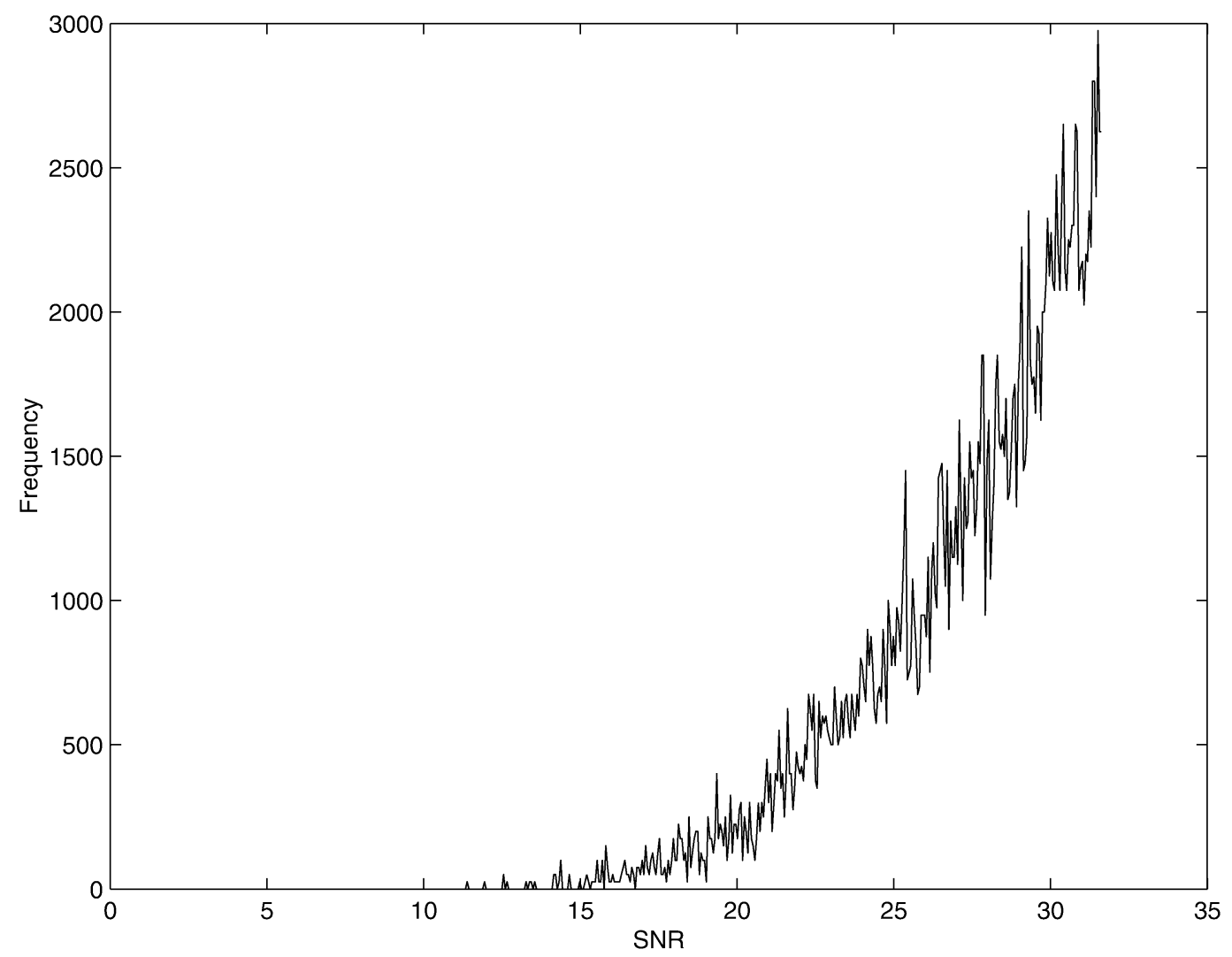

Fig. 3. The number of times that each user with the corresponding SNR is chosen for 50000 iterations with $M=1$ and $n=500$.

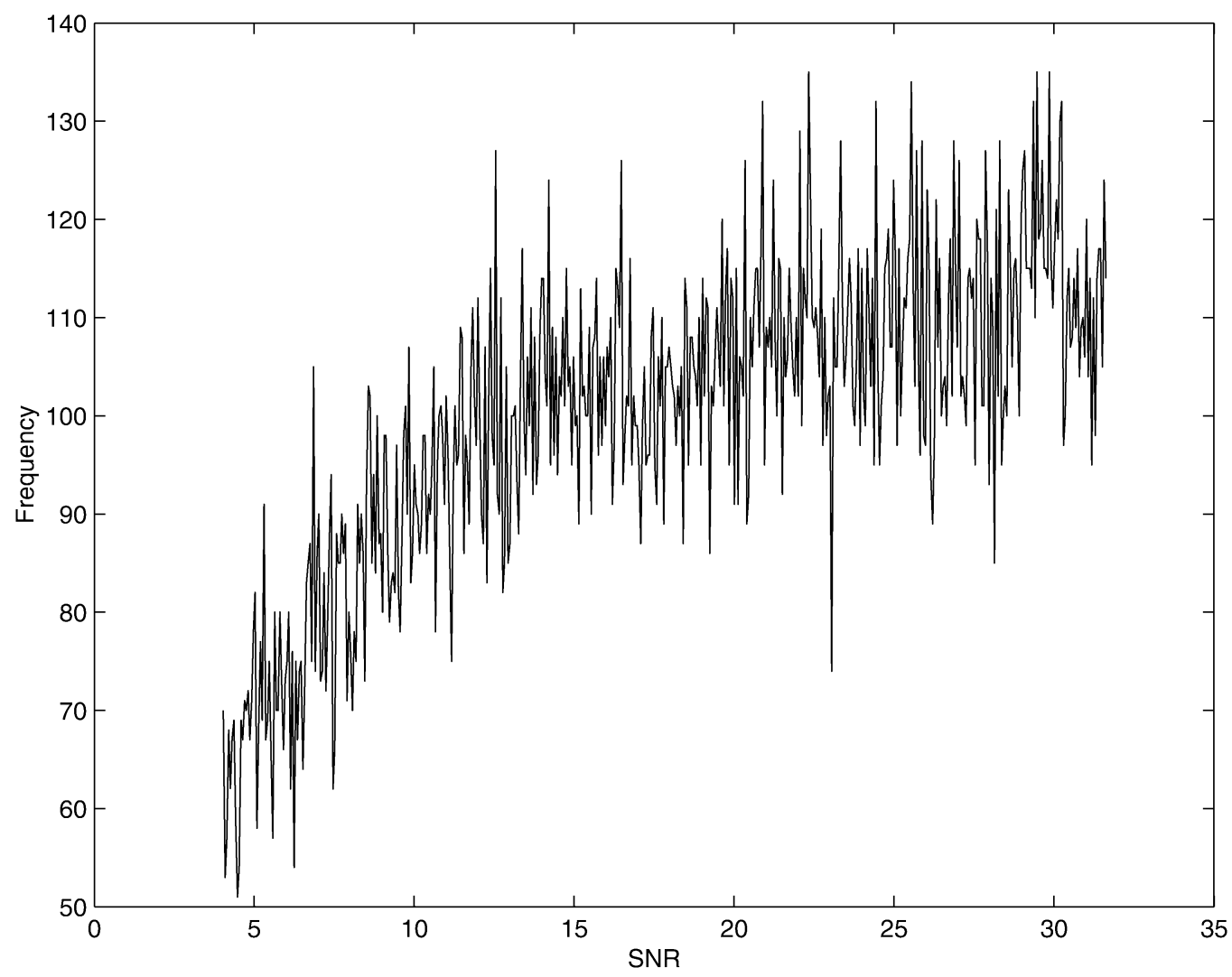

Fig. 4. The number of times that each user with the corresponding SNR is chosen for 10000 iterations with $M=5$ and $n=100$. 
is sufficiently large. This problem has been addressed in several papers and books (see, e.g., [17], [19], and references therein). It is known that for an arbitrary distribution, the density of the maximum does not necessarily have a limit as $n$ goes to infinity. In [17], necessary and sufficient conditions for the existence of a limit for the distribution of the maximum is established.

In what follows, Theorem A.1 presents all possible limiting distributions for the cumulative distribution of the maximum of $n$ i.i.d. random variables. Theorem A. 2 focuses on the class of distributions that are of interest in this paper and establishes the convergence rate to the limiting distribution. Finally, using Theorem A.2, we deduce Corollary A.1, which is the main result.

Theorem A.1: (Gnedenko, 1947) Let $x_{1}, \ldots, x_{n}$ be a sequence of i.i.d. random variables and

$$
x_{\max }=\max \left(x_{1}, \ldots, x_{n}\right) .
$$

Suppose that for some sequences $\left\{a_{n}>0\right\},\left\{b_{n}\right\}$ of real constants, $a_{n}\left(x_{\max }-b_{n}\right)$ converges in distribution to a random variable with distribution function $G(x)$. Then $G(x)$ must be one of the following three types:

$$
\begin{array}{rlrl}
\text { i) } & G(x) & =e^{-e^{-x}} & \\
\text { ii) } G(x) & =e^{-x^{-\alpha} u(x),} & & \alpha>0 \\
\text { iii) } & G(x) & =\left\{\begin{array}{lll}
e^{-(-x)^{\alpha}}, & \alpha>0 & x \leq 0 \\
1, & & x \geq 0 .
\end{array}\right.
\end{array}
$$

where $u(\cdot)$ is the step function.

Proof: Refer to [17], [23].

It turns out that the class of distribution functions we encounter in this paper are of type $i$. Therefore, we further look into sufficient conditions on the distribution of $x_{i}$ such that the distribution of the maximum is of type $i$.

We shall need the following definitions: let $x_{i}$ 's be positive random variables with continuous and strictly positive distribution function $f_{X}(x)$ for $x>0$ and cdf of $F_{X}(x)$, and define the growth function as $g_{X}(x)=\frac{1-F_{X}(x)}{f_{X}(x)}$. Further define $u_{n}$ to be the unique solution to

$$
1-F_{X}\left(u_{n}\right)=\frac{1}{n}
$$

(note that $u_{n}$ is unique due to the fact that $F_{X}(\cdot)$ is continuous and strictly increasing for $x \geq 0$ ). We now state the following result from [18].

Theorem A.2: (Uzgoren, 1956) Let $x_{1}, \ldots, x_{n}$ be a sequence of i.i.d. positive random variables with continuous and strictly positive pdf $f_{X}(x)$ for $x>0$ and cdf of $F_{X}(x)$. Let also $g_{X}(x)$ be the growth function. Then if $\lim _{x \rightarrow \infty} g(x)=c>0$, then

$$
\begin{gathered}
\log \left\{-\log F^{n}\left(u_{n}+u g\left(u_{n}\right)\right)\right\}=-u-\frac{u^{2} g^{\prime}\left(u_{n}\right)}{2 !}- \\
\cdots-\frac{u^{m} g^{(m)}\left(u_{n}\right)}{m !}+O\left(\frac{e^{-u+O\left(u^{2} g^{\prime}\left(u_{n}\right)\right)}}{n}\right)
\end{gathered}
$$

where $u_{n}$ is as defined in (A1).

Proof: Refer to the proof of [18, eq. (19)].

Consider, for example, a $\chi^{2}(2)$ random variable with $f(x)=$ $e^{-x} u(x)$. Then, it is quite easy to see that $g(x)=1, u_{n}=$ $\log n$, and all the derivatives of $g(x)$ are zero. Then, Theorem A. 2 simplifies to

$$
\operatorname{Pr}\left\{\max x_{i} \leq \log n+u\right\} \rightarrow e^{-e^{-u+o\left(\frac{e^{-u}}{n}\right)}} .
$$

Letting $u=\log \log n$ and $u=-\log \log n$ and using (A3) and (A2), we can easily show that

$\operatorname{Pr}\left\{\log n-\log \log n \leq \max x_{i} \leq \log n+\log \log n\right\}$

$$
\geq 1-O(1 / \log n) \text {. }
$$

Imposing a constraint on the derivatives of the growth function, we can use Theorem A.2 to state the following corollary which is used throughout the paper.

Corollary A.1: Let $x_{1}, \ldots, x_{n}$ be as defined in Theorem A.2. If $u_{n}=O(\log n)$ and $g(x)$ is such that $\lim _{x \rightarrow \infty} g(x)=c>0$ and $g^{(m)}\left(u_{n}\right)=O\left(1 / u_{n}^{m}\right)$, then

$\operatorname{Pr}\left\{u_{n}-c \log \log n \leq \max x_{i} \leq u_{n}+c \log \log n\right\}$

$$
\geq 1-O\left(\frac{1}{\log n}\right) \text {. }
$$

Proof: Since the distribution of $x_{i}$ 's satisfies the conditions of Theorem A.2, and $g\left(u_{n}\right)=c+o(1)$, we can choose $u=\log \log n$ and write the expansion of the distribution of $\max x_{i}$ as

$$
\begin{aligned}
\operatorname{Pr}\left\{\max x_{i} \leq u_{n}+c \log \log n\right\} & =F^{n}\left(u_{n}+c \log \log n\right) \\
& =e^{-e^{-\log \log n+o}\left(\frac{\log ^{2} \log n}{\log n}\right)} \\
& =1-O\left(\frac{1}{\log n}\right)
\end{aligned}
$$

where we used the identity $e^{x}=1+O(x)$ for small $x$ and also we used the fact that $u_{n}=O(\log n)$. Similarly, we may write

$$
\begin{aligned}
\operatorname{Pr}\left\{\max x_{i} \leq u_{n}-c \log \log n\right\} & =e^{-\log n(1+O(\log \log n / \log n))} \\
& =O\left(\frac{1}{n}\right) .
\end{aligned}
$$

Combining (A7) and (A6) completes the proof for this corollary.

Example 1: Suppose the $x_{i}$ 's have a $\chi^{2}(2 m)$ distribution and we apply Corollary A.1 to obtain the asymptotic behavior of $\max x_{i}$. We can write $g(x)$ as

$$
\begin{aligned}
g(x) & =\frac{1-F(x)}{f(x)}=\frac{(m-1) ! e^{-x} \sum_{i=0}^{m-1} \frac{x^{i}}{i !}}{e^{-x} x^{m-1}} \\
& =(m-1) ! \sum_{i=0}^{m-1} \frac{x^{i-(m-1)}}{i !} .
\end{aligned}
$$

In order to find $u_{n}$, we use the asymptotic expansion of the incomplete Gamma function to get [21]

$$
\begin{aligned}
1-F\left(u_{n}\right)= & \Gamma\left(m, u_{n}\right)=u_{n}^{m-1} e^{-u_{n}}\left(1+O\left(1 / u_{n}\right)\right) \\
= & \frac{1}{n} \Longrightarrow u_{n}=\log n+(m-1) \log \log n \\
& +O(\log \log \log n) .
\end{aligned}
$$


We can also observe that $g^{(m)}\left(u_{n}\right)=O\left(1 / u_{n}^{m}\right)$. Therefore, the maximum value of $n$ i.i.d. $\chi^{2}(2 m)$ random variables satisfies

$$
\begin{array}{r}
\operatorname{Pr}\{\log n+(m-2) \log \log n+O(\log \log \log n) \\
\left.\leq \max _{1 \leq i \leq n} x_{i} \leq \log n+m \log \log n+O(\log \log \log n)\right\} \\
>1-O\left(\frac{1}{\log n}\right) .
\end{array}
$$

\section{APPENDIX B \\ PROOF OF LEMMA 1}

In this appendix, we prove that with full knowledge of the channel at the transmitter, the maximum sum-rate throughput scales like $M \log \log n$ as $n$ is going to infinity and $M$ is fixed. The sum rate in MIMO broadcast channel has been recently addressed by several others [6]-[8]. The asymptotic behavior of the sum rate is also analyzed for the case where $M / n$ is a constant greater than one and $n$ is growing to infinity [20]. Using the duality between the broadcast channel and MAC, the sum rate of MIMO BC, $R^{D P}$ is equal to [7], [8]

$R^{D P}=E\left\{\max _{\left\{P_{1}, \ldots, P_{n}, \sum P_{i}=M \rho\right\}} \log \operatorname{det}\left(1+\sum_{i=1}^{n} H_{i}^{*} P_{i} H_{i}\right)\right\}$

where $H_{i}$ are $1 \times M$ channel matrices with i.i.d. $C N(0,1)$ distributions, $P_{i}$ is the optimal power scheduling, and $M \rho$ is the total transmit power.

Now we can prove Lemma 1, by using the inequality

$$
\operatorname{det}(A) \leq\left(\frac{\operatorname{tr}(A)}{M}\right)^{M}
$$

where $A$ is an $M \times M$ matrix. Therefore, (B1) can be written as (B2)-(B5) at the bottom of the page, where we used $\operatorname{tr}(A B)=$ $\operatorname{tr}(B A)$ in the second equality and $\operatorname{tr}\left(H_{i}^{*} H_{i}\right)$ has $\chi^{2}(2 M)$ distribution. It is shown in Example 1 of Appendix A that with high probability the maximum behaves like $\log n+O(\log \log n)$. Therefore,

$$
R \leq M \log (1+\rho \log n+O(\log \log n)) .
$$

One way to prove that $M \log \log n$ is achievable, is to use our random beamforming scheduling which is shown to achieve $M \log \log n$ in Theorem 1 .
Therefore, we get $R \geq M \log (1+\rho \log n+O(\log \log n))$ which completes the achievability part of the proof. The lemma follows by using the upper and lower bounds for the sum rate capacity.

\section{APPENDIX C \\ PROOF OF (36)}

In this appendix, we compute a lower bound for $\operatorname{Pr}\{B \mid A\}$ where $A$ is the event that for all $m, c-\epsilon \leq \max _{1 \leq i \leq n} \operatorname{SINR} \leq c$, and $B$ is the event that each user can be the maximum for at most one signal $s_{m}$. Let us assume $p_{m}=\arg \max _{i} \operatorname{SINR}_{i, m}$. Therefore, we can upper-bound $1-\operatorname{Pr}\{B \mid A\}$ by the probability of the event that there exists an index $p_{m}$ such that the corresponding user is the maximum for at least two signals $s_{m_{1}}$ and $s_{m_{2}}$. Since this event is conditioned on the event $A$, both the maxima should be between $c$ and $c-\epsilon$ and clearly one of them should be $\max _{1 \leq m \leq M} \operatorname{SINR}_{p_{1}, m}$. Therefore,

$$
\begin{aligned}
& 1-\operatorname{Pr}\{B \mid A\} \\
& \leq \operatorname{Pr}\left\{\exists p \in\left\{p_{1}, \ldots, p_{M}\right\}: c-\epsilon\right. \\
& \left.\quad \leq \operatorname{SINR}_{p_{1}, m_{1}}, \max _{1 \leq m \leq M} \operatorname{SINR}_{p_{1}, m} \leq c\right\} \\
& \leq M \operatorname{Pr}\left\{c-\epsilon \leq \mathrm{SINR}_{1, m_{1}}, \max _{1 \leq m \leq M} \operatorname{SINR}_{1, m} \leq c\right\}
\end{aligned}
$$

where we used the union bound and the fact that all SINRs have the same distribution over $i$, and $m_{1}$ is not the index corresponding to the maximum SINR over $m$. In order to compute the probability in $(\mathrm{C} 1)$, we define the random variables $\beta_{m}=\left|h_{1} \phi_{m}\right|^{2}$ for $m=1, \ldots, M$, and let $r=\arg \max \beta_{i}$. Therefore, we want to compute the probability that

$$
c \geq \max _{1 \leq m \leq M} \operatorname{SINR}_{1, m}=\frac{\max \beta_{i}}{\frac{1}{\rho}+\sum_{i \neq r}^{M} \beta_{i}}=\frac{\max \beta_{i}}{\frac{1}{\rho}+\beta_{m_{1}}+D}
$$

and that there exists $m_{1} \in\{1, \ldots, M\}$ and $m_{1} \neq r$ such that

$$
c-\epsilon \leq \operatorname{SINR}_{1, m_{1}}=\frac{\beta_{m_{1}}}{\frac{1}{\rho}+\max \beta_{i}+D}
$$

where

$$
D=\sum_{i=1}^{M} \beta_{i}-\max \beta_{i}-\beta_{m_{1}} .
$$

$$
\begin{aligned}
R^{D P} & \leq M E\left\{\max _{\left\{P_{1}, \ldots, P_{n}, \sum P_{i}=M \rho\right\}} \log \left(1+\frac{\sum \operatorname{tr}\left(H_{i}^{*} P_{i} H_{i}\right)}{M}\right)\right\} \\
& =M E\left\{\max _{\left\{P_{1}, \ldots, P_{n}, \sum P_{i}=M \rho\right\}} \log \left(1+\frac{\sum \operatorname{tr}\left(H_{i} H_{i}^{*}\right) P_{i}}{M}\right)\right\} \\
& \leq M E\left\{\max _{\left\{P_{1}, \ldots, P_{n}, \sum P_{i}=M \rho\right\}} \log \left(1+\frac{\max _{1 \leq i \leq n} \operatorname{tr}\left(H_{i} H_{i}^{*}\right) \sum P_{i}}{M}\right)\right\} \\
& =M E\left\{\log \left(1+\rho \max _{1 \leq i \leq n} \operatorname{tr}\left(H_{i} H_{i}^{*}\right)\right)\right\}
\end{aligned}
$$




$$
\begin{aligned}
1-\operatorname{Pr}\{B \mid A\} & \leq M \operatorname{Pr}\left\{\exists m_{1} \in\{1, \ldots, M\}, m_{1} \neq r: \beta_{m_{1}} \geq\left(1-\frac{\epsilon}{c}\right) \max \beta_{i}\right\} \\
& =M \int_{0}^{\infty}\left(\begin{array}{c}
M \\
2
\end{array}\right) \operatorname{Pr}\left\{a\left(1-\frac{\epsilon}{c}\right) \leq \beta_{1} \leq a\right\}^{2}\left(\operatorname{Pr}\left\{\beta_{1}<a\right\}\right)^{M-2} d a \\
& =\frac{M^{2}}{2}(M-1) \int_{0}^{\infty}\left(e^{-\left(1-\frac{\epsilon}{c}\right) a}-e^{-a}\right)^{2}\left(1-e^{-a}\right)^{M-2} d a \\
& =\frac{M^{2}}{2}(M-1) \int_{0}^{1}\left(u^{-\epsilon}-1\right)^{2} u(1-u)^{M-2} d u
\end{aligned}
$$

Equations (C2) and (C3) imply that $\beta_{m_{1}} \geq\left(1-\frac{\epsilon}{c}\right) \max \beta_{i}$. This probability can be computed by integrating over $a$ the probability that there are at least two $\beta_{i}$ 's in the region $\left[a\left(1-\frac{\epsilon}{c}\right), a\right]$ and all the $\beta_{i}$ 's are less than $a$. Therefore, we get $(\mathrm{C} 4)$ at the top of the page, where in the second step we use the fact that two $\beta_{i}$ 's must be large (in fact, in the region $\left[a\left(1-\frac{\epsilon}{c}\right), a\right]$ ) and $\beta_{i}$ 's are i.i.d random variables. In order to compute $(\mathrm{C} 4)$, we define the function

$$
T(\epsilon)=\int_{0}^{1} u^{1-\epsilon-x}(1-u)^{M-2} d u .
$$

Clearly, the integral in (C4) can be written as $T(\epsilon)+T(-\epsilon)-$ $2 T(0)$. By mean value theorem, we can use the Taylor expansion of the integral to get

$$
\begin{aligned}
\int_{0}^{1}\left(u^{-\epsilon}-1\right)^{2} u(1-u)^{M-2} d u & =T(\epsilon)+T(-\epsilon)-2 T(0) \\
& =T^{\prime \prime}(\zeta) \epsilon^{2}
\end{aligned}
$$

where $-\epsilon \leq \zeta \leq \epsilon$. Now we can write the second derivative of $T(\zeta)$ as

$$
\begin{aligned}
T^{\prime \prime}(\gamma) & =\int_{0}^{1}(\log u)^{2} u^{1-\epsilon-\zeta}(1-u)^{M-2} d u \\
& \leq \int_{0}^{1}(\log u)^{2} u^{0.9}(1-u)^{M-2} d u
\end{aligned}
$$

where we used the fact that $\epsilon$ is very small and $-\epsilon \leq \zeta \leq \epsilon$. We know that $u^{0.4}(\log u)^{2}$ is a bounded function for $u \in[0,1]$, therefore,

$$
\begin{aligned}
T^{\prime \prime}(\gamma) & \leq O\left(\int_{0}^{1} u^{0.5}(1-u)^{M-2} d u\right) \\
& =O\left(\frac{\Gamma(M-1) \Gamma(1.5)}{\Gamma(M+0.5)}\right) \\
& =O\left(\frac{1}{M^{1.5}}\right)
\end{aligned}
$$

where we used the asymptotic expansion of the Gamma functions [21]. Replacing (C7) in (C5) and then into (C4), we get

$$
1-\operatorname{Pr}\{B \mid A\} \leq O\left(\epsilon^{2} M^{1.5}\right)=O\left(\frac{(\log \log n)^{2}}{\sqrt{\log n}}\right) \text {. }
$$

\section{REFERENCES}

[1] E. Telatar, "Capacity of multi-antenna Gaussian channel," Europ. Trans. Telecommun., vol. 10, pp. 585-595, Nov. 1999.

[2] G. J. Foschini and M. J. Gans, "On limits of wireless communications in a fading environment when using multiple antennas," Wireless Personal Commun., vol. 6, pp. 311-335, Mar. 1998.
[3] L. Zheng and D. N. C. Tse, "Communications on the Grassman manifold: A geometric approach to the noncoherent multiple-anetnna channel," IEEE Trans. Inf. Theory, vol. 48, no. 2, pp. 359-384, Feb. 2002.

[4] B. Hassibi and T. L. Marzetta, "Multiple-antennas and isotropically random unitary inputs: The received signal density in closed form," IEEE Trans. Inf. Theory, vol. 48, no. 6, pp. 1473-1484, Jun. 2002.

[5] B. Hochwald, T. Marzetta, and V. Tarokh, "Multi-antenna channel-hardening and its implications for rate feedback and scheduling," IEEE Trans. Inf. theory, vol. 50, no. 9, pp. 1893-1909, Sep. 2004.

[6] G. Caire and S. Shamai (Shitz), "On the achievable throughput of a multiantenna Gaussian broadcast channel," IEEE Trans. Inf. Theory, vol. 49, no. 7, pp. 1691-1706, Jul. 2003.

[7] P. Viswanath and D. N. C. Tse, "Sum capacity of the vector Gaussian broadcast channel and downlink-uplink duality," IEEE Trans. Inf. theory, vol. 49, no. 8, pp. 1912-1921, Aug. 2003.

[8] S. Vishwanath, N. Jindal, and A. Goldsmith, "Duality, achievable rates and sum rate capacity of Gaussian MIMO broadcast channel," IEEE Trans. Inf. Theory, vol. 49, no. 10, pp. 2658-2668, Oct. 2003.

[9] H. Weingarten, Y. Steinberg, and S. Shamai (Shitz), "The capacity region of the Gaussian MIMO broadcast channel," in Proc. IEEE Int. Symp. Information Theory, Chicago, IL, Jun./Jul. 2004, p. 174.

[10] W. Yu and J. M. Cioffi, "Trellis precoding for the broadcast channel," in Proc. IEEE Global Communications Conf., vol. 2, San Antonio, TX, 2001, pp. 1344-1348.

[11] R. Zamir, S. Shamai (Shitz), and U. Erez, "Nested linear/lattice codes for structured multiterminal binning," IEEE Trans. Inf. Theory, vol. 48, no. 6, pp. 1250-1277, Jun. 2002.

[12] C. B. Peel, B. Hochwald, and A. L. Swindlehurst, "A vector perturbation technique for near capacity multi-antenna multi-user communication-Part I: Channel inversion and regularization," IEEE Trans. Commun., submitted for publication.

[13] P. Viswanath, D. N. C. Tse, and R. Laroia, "Opportunistic beamforming using dump antennas," IEEE Trans. Inf. Theory, vol. 48, no. 6, pp. 1277-1294, Jun. 2002.

[14] M. Sharif and B. Hassibi, "Scaling laws of sum rate using time-sharing, DPC, and beamforming for MIMO broadcast channels," in Proc. IEEE Int. Symp. Information Theory, Chicago, IL, Jun./Jul. 2004, p. 175.

[15] A. Goldsmith, S. A. Jafar, N. Jindal, and S. Vishwanath, "Capacity limits of MIMO channels," IEEE J. Sel. Areas. Commun., vol. 21, no. 5, pp. 684-702, Jun. 2003.

[16] J. C. Roh and B. Rao, "Multiple antenna channels with partial feedback," in Proc. IEEE Int. Conf. Communications, vol. 5, Anchorage, AK, 2003, pp. 3195-3199.

[17] M. R. Leadbetter, "Extreme value theory under weak mixing conditions," in Studies in Probability Theory, MAA Studies in Mathematics, 1978, pp. 46-110.

[18] N. T. Uzgoren, "The asymptotic developement of the distribution of the extreme values of a sample," in Studies in Mathematics and Mechanics Presented to Richard von Mises. New York: Academic, 1954, pp. 346-353.

[19] H. A. David, Order Statistics. New York: Wiley, 1970.

[20] B. Hochwald and S. Viswanath, "Space time multiple access: Linear growth in the sum rate," presented at the 40th Annu. Allerton Conf., Monticello, IL, 2002.

[21] I. S. Gradshteyn and I. M. Ryzhik, Table of Integrals, Series, and Products. London, U.K.: Academic, 1965.

[22] A. Edelman, "Eigenvalues and condition numbers of random matrices," Ph.D. dissertation, MIT, Cambridge, MA, 1989.

[23] M. R. Leadbetter and H. Rootzen, "Extremal theory for stochastic processes," Ann. Probab., vol. 16, pp. 431-478, 1988. 